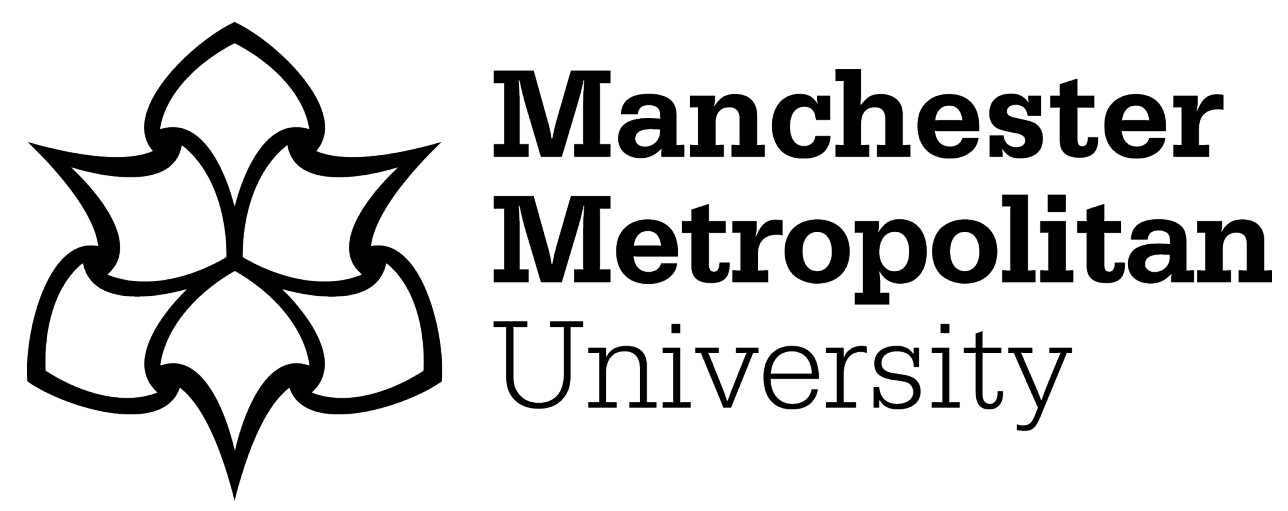

Hassan, Haseeb, Bashir, Ali Kashif, Ahmad, Muhammad, Menon, Varun G, Afridi, Imran Uddin, Nawaz, Raheel ORCID logoORCID: https://orcid.org/0000-0001-9588-0052 and Luo, Bin (2021) Real-time image dehazing by superpixels segmentation and guidance filter. Journal of RealTime Image Processing, 18 (5). pp. 1555-1575. ISSN 1861-8200

Downloaded from: https://e-space.mmu.ac.uk/625385/

Version: Accepted Version

Publisher: Springer Science and Business Media LLC

DOI: https://doi.org/10.1007/s11554-020-00953-4

Please cite the published version 


\section{Real-time image dehazing by superpixels segmentation and guidance filter}

\section{Haseeb Hassan, Ali Kashif Bashir,}

Muhammad Ahmad, Varun G. Menon, Imran Uddin Afridi, Raheel Nawaz \&

\section{Bin Luo}

Journal of Real-Time Image

Processing

ISSN 1861-8200

J Real-Time Image Proc

DOI 10.1007/s11554-020-00953-4 
Your article is protected by copyright and all rights are held exclusively by SpringerVerlag GmbH Germany, part of Springer Nature. This e-offprint is for personal use only and shall not be self-archived in electronic repositories. If you wish to self-archive your article, please use the accepted manuscript version for posting on your own website. You may further deposit the accepted manuscript version in any repository, provided it is only made publicly available 12 months after official publication or later and provided acknowledgement is given to the original source of publication and a link is inserted to the published article on Springer's website. The link must be accompanied by the following text: "The final publication is available at link.springer.com". 


\title{
Real-time image dehazing by superpixels segmentation and guidance filter
}

\author{
Haseeb Hassan ${ }^{1} \cdot$ Ali Kashif Bashir ${ }^{2} \cdot$ Muhammad Ahmad $^{3,4} \cdot$ Varun G. Menon $^{5} \cdot$ Imran Uddin Afridi $^{6}$. \\ Raheel Nawaz ${ }^{7}$ Bin Luo ${ }^{1}$
}

Received: 8 November 2019 / Accepted: 19 February 2020

○) Springer-Verlag GmbH Germany, part of Springer Nature 2020

\begin{abstract}
Haze and fog had a great influence on the quality of images, and to eliminate this, dehazing and defogging are applied. For this purpose, an effective and automatic dehazing method is proposed. To dehaze a hazy image, we need to estimate two important parameters such as atmospheric light and transmission map. For atmospheric light estimation, the superpixels segmentation method is used to segment the input image. Then each superpixel intensities are summed and further compared with each superpixel individually to extract the maximum intense superpixel. Extracting the maximum intense superpixel from the outdoor hazy image automatically selects the hazy region (atmospheric light). Thus, we considered the individual channel intensities of the extracted maximum intense superpixel as an atmospheric light for our proposed algorithm. Secondly, on the basis of measured atmospheric light, an initial transmission map is estimated. The transmission map is further refined through a rolling guidance filter that preserves much of the image information such as textures, structures and edges in the final dehazed output. Finally, the haze-free image is produced by integrating the atmospheric light and refined transmission with the haze imaging model. Through detailed experimentation on several publicly available datasets, we showed that the proposed model achieved higher accuracy and can restore high-quality dehazed images as compared to the state-of-the-art models. The proposed model could be deployed as a real-time application for real-time image processing, real-time remote sensing images, real-time underwater images enhancement, video-guided transportation, outdoor surveillance, and autodriver backed systems.
\end{abstract}

Keywords Dehazing $\cdot$ Defogging $\cdot$ Real-time remote sensed images haze removal $\cdot$ Real-time underwater images enhancement $\cdot$ Statistical method of dark channel prior $\cdot$ Superpixels segmentation

Bin Luo

luobin@ahu.edu.cn

Haseeb Hassan

haseeb87@yahoo.com

Muhammad Ahmad

mahmad00@gmail.com

1 School of Computer Science and Technology, Anhui University, Hefei, China

2 School of Computing, Mathematics, and Digital Technology, Manchester Metropolitan University, Manchester, UK

3 Dipartimento di Matematica e Informatica-MIFT, University of Messina, 98121 Messina, Italy
4 Advanced Image Processing Research Lab (AIPRL), Department of Computer Engineering, Khwaja Fareed University of Engineering and Information Technology, Rahim Yar Khan, Pakistan

5 Department of Computer Science and Engineering, SCMS School of Engineering and Technology, Ernakulam 683576, India

6 Department of Computer Science, COMSATS University, Islamabad, Pakistan

7 Department of Operations, Technology, Events and Hospitality Management, Manchester Metropolitan University, Manchester, UK 


\section{Introduction}

Acquiring high-quality images has rapidly increased to meet practical applications such as vision systems, global positioning system (GPS), and remote sensing. However, such images have bad quality due to haze, fog, dust particles and water droplets, which cannot be used for realtime applications due to the attenuation of the flux radiant energy received by a sensor along the line of sight. Moreover, the light received by a sensor is a mixture of different things or qualities (dust, smoke, and dry particles) [1]. Likewise, haze also causes color distortion of the image. The aforesaid discussion reveals that defogging and dehazing play an important role and provide a faster aid for real-time applications such as video-guided transportation [2-4], outdoor surveillances [5-7], for analyzing real-time remote sensing images [8-11], and the auto-driver backed systems [12-14]. Moreover, the dehazing algorithms can be extended for real-time underwater images enhancement. After removing haze, the restored image appears better and the appearance of the objects looks clear, which provide easy understanding for vision systems, satellites imagery, and surveillance systems. Haze elimination is an ill-posed process and much-needed research for photography, computer vision, and real-time image processing applications.

Haze removal methods can be classified into three main categories. The first one is enhancement [15-18], the second one is image restoration [19-24] which are based on a physical model [25] and the third one is fusionbased methods [26-28]. The enhancement-based methods improve the visual quality and contrast of the image but do not remove the full haze [7]. On the other hand, in the image restoration-based methods, a physical model is involved for degradation of the hazy image, where the lost information is compensated with the inversion algorithm, which has a natural effect of dehazing. Our method falls at the restoration side so we will mainly focus on restorationbased methods. The image restoration-based techniques provide the analysis of image degradation and imaging mechanism where the scene can be recovered by inverse transformation. The restoration-based methods divided into three subcategories: (1) multiple images-based dehazing, (2) additional information-based dehazing, and (3) prior knowledge-based dehazing. Multiple images-based dehazing techniques further contain weather conditions and polarization-based dehazing.

In polarization-based methods [23, 29] the haze removed from multiple images captured at different degrees of polarization. For instance scheme [23] presented an approach based on air-light scattering. Images were taken on dissimilar orientations through the polarizer. In this method, a depth map of the scene and information of the atmospheric particles were yielded, which improved the contrast of the scene and the correction of color. The method proposed in [29] blindly separated the air-light radiance from the object signal. Since the air-light causes contrast degradation. It worked even without the existence of the sky region in the field of view (FOV) and automatically determined the parameters for separation. This automatic separation process reduced the user interaction for dehazing.

Likewise, the works $[21,22,30,31]$ have more constraints obtained from the same scene under different weather conditions. In [21] maximum and minimum depths of field are specified artificially to obtain approximate depth information and recovered a clear image based on the physical model. The method proposed in [22] is based on atmospheric optics identify some important aspects of bad weather. These effects considered as advantageous for dehazing. This work also demonstrated the atmosphere modalities as information from scene point to the observer and further developed models to recover relevant scene properties. P. Tavallai et al [30] used the statistical local luminance features to build a fast and robust skin detector based on Cascaded AdaBoost for face detection and recognition issues. Another physics-based model is proposed in [31] which defined scene appearances in bad weather conditions. In bad weather, the dynamic intensities of scene points provide constraints to detect depth discontinuities in the scene. Additionally, it computed the scene structure and a scene contrast was restored without any prior scene structure.

Nowadays, single image dehazing gained much attention due to its stronger prior and assumption [32]. Single image dehazing has auxiliary branches like direct air-light estimation [32, 33], anisotropic diffusion [34], and contrast maximization-based $[35,36]$ techniques. A statistical-based scheme proposed in [32] referred to as dark channel prior (DCP) method. The suggested algorithm initially applied the minimum operator and obtained the lowest pixel intensities called a dark channel, where the air-light is estimated directly from the highest pixels. After atmospheric light estimation, the initial transmission was obtained and further refined by the soft matting interpolation method. Finally, the radiance produced from the estimated atmospheric light and transmission map. The work proposed in [33] targeted the restored image and maximized its contrast which is based on the assumption that the haze-free image has higher contrast than the haze image. However, the method has still halo artifacts in the final output map. In the anisotropic diffusion method [34] the post- and pre-processing steps require, where histogram equalization and histogram stretching are used. The aforesaid scheme worked well for both color and gray-scale foggy images. 
The work [35] is based on scattered light elimination to increase the scene visibility and recovered a haze-free FOV. In which, a refined image formation model adopted for surface shading. According to the image formation model, the image was broken into different regions of constant albedo where an additional constraint is used to resolve the air-light ambiguity. The constraint imposes local statistical un-correlation for surface shading and medium transmission. Finally, the graphical model is used to propagate the derived pixels. The results were convincing; however, the sky region in the haze image limits the performance. The work [36] presented a visibility restoration-based model for single image dehazing based on filtering approach. The median filter is used to estimate the atmospheric transmission, and further, a tone map is applied to get the dehaze image with the limitation to halo effects.

To overcome such limitations, a color attenuation prior method was proposed in [37], in which the depth information is recovered through a linear model. From the depth information, the transmission is estimated and the scene radiance is restored through the atmospheric scattering model which produced better dehaze results. Meanwhile, the works [38, 39] demonstrate a color lines and regularization schemes to dehaze the haze image. Another way around, the works $[40,41]$ used a cost function which relied on contrast and the amount of lost information and adaptive wiener filtering for dehazing. A fusion-based method was proposed in [26] in which an image is composed of separate layers such as scene albedo and scene depth. The depth information was computed using factorial Markov random field; however, the contrast in the resultant image was too saturated. Similarly, a fusion-based work proposed in [27] modeled the image with FMRF where an image is factorized into scene albedo and depth. In this technique the key insight is that both scene albedo and depth have important structural information which are influential to the resulting hazy image. Finally a single foggy image is factorized by a canonical expectation maximization algorithm. A technique proposed in [28] fused two coarse transmission maps using the dark channel prior (DCP). This method worked well in terms of computational speed and applicable to the real-time applications.

This work focused on the problems persist in given methods [32, 35-37, 39, 42]. The work [32] provided a new direction of dark channel prior; however, it has also some limitations such as over-saturation and halo effects in the final output map. Moreover, this method uses soft matting to refine the transmission map which is computationally an expensive task. Later on, a work proposed in [42] is integrated with the DCP method [32]. The guided filtering solved the computational complexity and preserved much of the edges information too. However, the over-saturation and halo effects still persist. Furthermore, the work [35] may get failed when the pre-assumptions void, hence unable to completely remove haze. Similarly, the work [36] tries to simplify the dehazing process; however, it is not vibrant approach due to the segregation of small edge regions. In addition to the above, the work [37] presents a robust haze removal method, both in terms of results and computations; however, its training procedure is complex due to the parameters which largely depend on the training data. Therefore, this work proposed a novel procedure consisting of superpixels masking and rolling guidance filter-based haze removal method which is in fact related with both statistical-based DCP [32] and guided filtering [42] methods. The proposed method comprises the following steps:

1. Air-light is estimated through superpixels segmentation.

2. The rolling guidance filter is adopted instead of softmatting and guided filter to refine the transmission map which preserves the edges, structure, and textures characteristics.

The rest of the paper is structured as follows: Sect. 2 presents the background and haze imaging model. Section 3 illustrates the proposed methodology. Section 4 describes the results and their discussion, and finally Sect. 5 concludes the paper with possible future research directions.

\section{Background}

\subsection{Haze imaging model}

The haze imaging model used in $[31,43,44]$ is illustrated in Fig. 1. The mathematical representation of the hazy image formation model is given as:

$I\left(x_{g}\right)=J\left(x_{g}\right) t\left(x_{g}\right)+A\left(1-t\left(x_{g}\right)\right)$

where $I$ is the haze image, $J$ is the scene radiance (haze-free image) and $x_{g}$ is the pixel location, whereas $A$ denotes the air-light and $t$ is the medium transmission which describes the portion of the light, which is not scattered and reaches the sensor. The term $J\left(x_{g}\right) t\left(x_{g}\right)$ denotes the direct attenuation [33] and the second part $A\left(1-t\left(x_{g}\right)\right)$ refers to air-light or atmospheric light $[29,33]$. The both terms also provide theoretical basis for blurred hazy images [45-48]. The transmission $t\left(x_{g}\right)$ in a homogeneous atmosphere and can be expressed as:

$t\left(x_{g}\right)=e^{-\beta d\left(x_{g}\right)}$ 

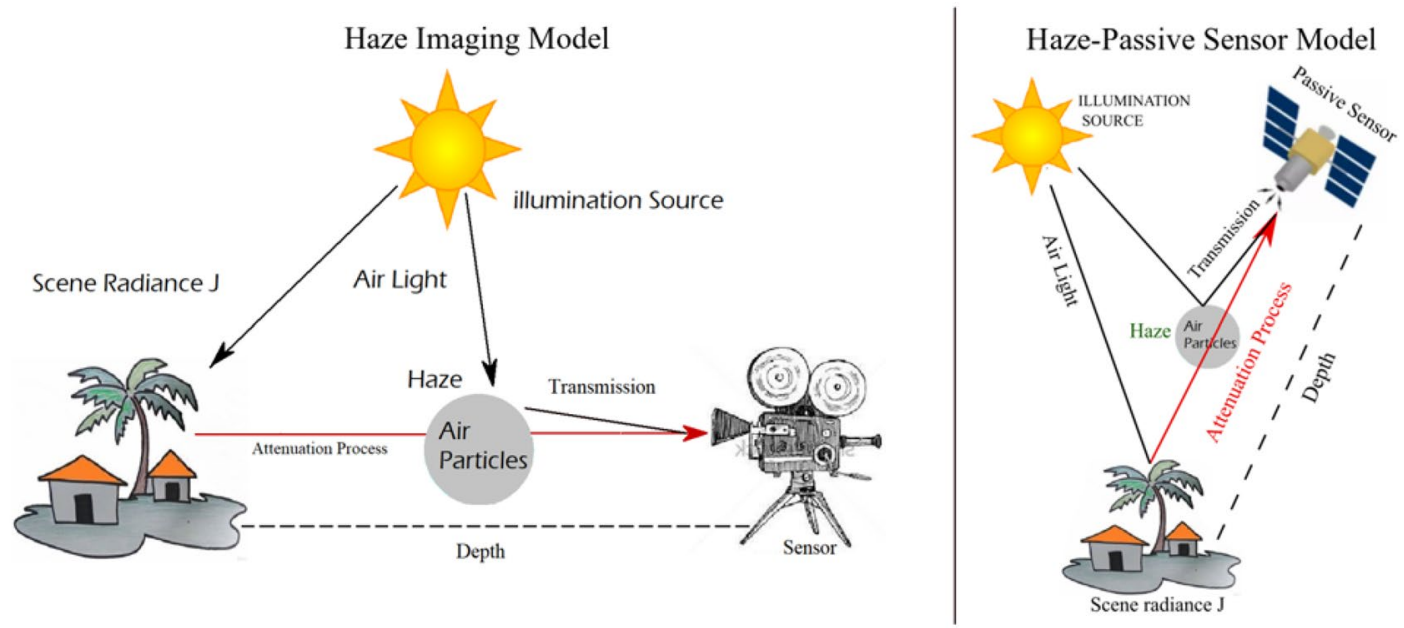

Fig. 1 On left the atmospheric scattering model suggests that the hazy imaging model scene consists of two main parts. One is the attenuation process which is reflected light from the scene surface to

where $\beta$ is scattering coefficient and $d$ is the scene depth. The $\beta=0$ in a clear weather means that $I \approx J$, however, when $\beta$ has some value it results in a hazy image. Besides, the transmission $t$ can be defined as the ratio of two line segments from Equation 1 and given as:

$t\left(x_{g}\right)=\frac{\left\|A-I\left(x_{g}\right)\right\|}{\left\|A-J\left(x_{g}\right)\right\|}=\frac{A^{c}-I^{c}\left(x_{g}\right)}{A^{c}-J^{c}\left(x_{g}\right)}$

where $C$ belongs to $r, g, b$ and is the color channel index. The discussed, degraded imaging model reveals that dehazing is all about the estimation of the air-light $A$, and transmission $t\left(x_{g}\right)$ through which the final haze-free image $J\left(x_{g}\right)$ can be restored from $I\left(x_{g}\right)$.

\subsection{Dark channel prior (DCP) theory}

The dark channel prior (DCP) method proposed in [32] is a statistical and simple yet effective method which explores the dark pixels phenomena to compute the thickness of the haze to recover a haze-free image. In DCP method [32] extensive experimentation is performed on thousands of outdoor hazy images which explored that at least one color channel has the lowest pixel intensities ignoring the sky region which tends to zeros and yielding a dark channel. These dark pixels appear due to the shadows, trees, plants, and some dark surfaces like stones and rocks. This statistical observation revealed that in the presence of haze, the air-light can alter the dark pixel values and provides a direct contribution to the values of dark pixels. Therefore, these dark pixels are vibrant clue to estimate haze transmission. Mathematically, the dark channel for an image is defined as: the sensor and the second one air-light scattering reaches to the sensor. At the right side, the haze imaging model when applied to a passive satellite sensor for defogging the satellite imagery

$J^{\mathrm{dark}}\left(x_{g}\right)=\min _{c \in\{r, g, b\}}\left(\min _{y \in \Omega\left(x_{g}\right)}\left(J^{c}(y)\right)\right) \rightarrow 0$

where $J^{c}$ is a color channel of $J$ and $\Omega\left(x_{g}\right)$ is a patch centering at $x_{g}$, and $\min _{c \in\{r, g, b\}}$ is the minimum operator which is applied to all color channels and selects the lowest pixels intensities. According to DCP [32], in haze-free image the intensity of $J^{\text {dark }}$ is low and has a tendency toward zero while ignoring the sky region. Therefore, $J^{\text {dark }}$ is demonstrated as a dark channel of $J$.

Apart from the success of DCP method [32], it has also some limitations such as the use of soft-matting which results in slow processing. Secondly, the production of oversaturation, distortion and halo effect in the final output map.

\section{Proposed method of superpixels masking and rolling guidance filter}

The proposed haze removal method overcomes the aforementioned limitations by first segmenting the outdoor input hazy image into superpixels by using the simple linear iterative clustering (SLIC) algorithm [49]. After superpixels computation, we extracted the most intense superpixel among the all computed superpixels which automatically selects the hazy region of the input hazy image. Therefore, we considered the extraction of the maximum intense superpixel as an air-light parameter for our proposed algorithm.

On the second stage, the transmission map is estimated from the air-light and dark channel. For the refinement of the transmission map further, a rolling guidance filter [50] is applied instead of guided filtering [42] to preserve more strong edges, better structure, and texture. Our two proposed 


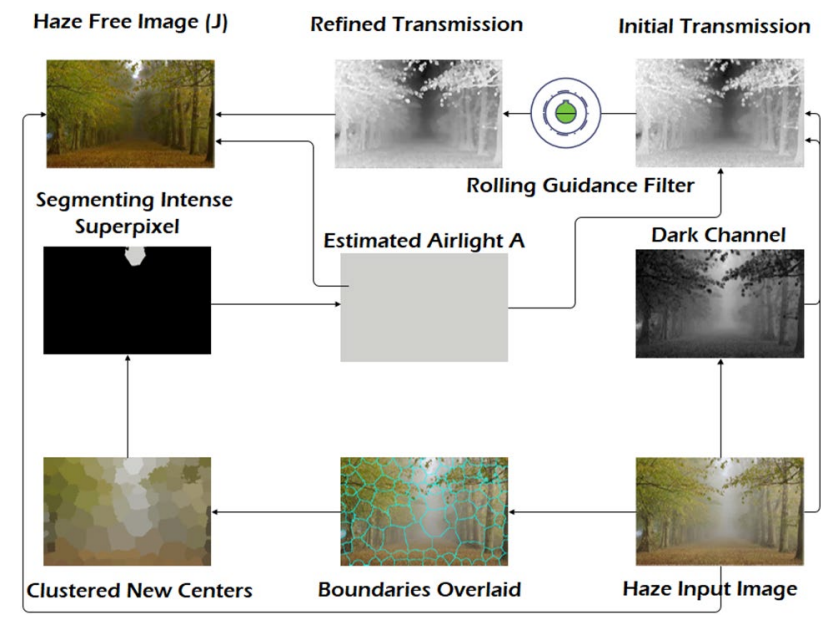

Fig. 2 The proposed algorithm. Where $I$ is an input haze image, initially the superpixel boundaries are overlaid. Next the pixels are re-clustered and new centers are defined. The maximum intense superpixel extracted from the image and we considered it as an atmospheric light parameter. Getting the air-light value, the transmission $t$ is estimated and rolling guidance filter is applied to refine the estimated transmission $t$. Finally, the scene radiance $J$ is recovered

novel settings of air-light estimation and transmission refinement help to obtain a better scene Radiance, i.e., the hazefree image. Figure 2 shows the important steps of our proposed method which are further explained in the following sections.

\subsection{Air-light estimation by superpixels segmentation}

Recently superpixels have drawn more attention due to its usefulness in computer vision applications. Many algorithms proposed which outputs compact superpixels according to user desire at low computational cost. However, Achanta et al. [49] proposed an effective simple linear iterative clustering (SLIC) algorithm for superpixels computation. In their method, a three-channel image is taken into account where the image pixels are considered as $N$ numbers. The amount of superpixels is defined by $K$. The average area of a superpixel is derived by this simple formula of $N / K$. A single superpixel is defined as:

$C_{k}=\left[R_{k}, G_{k}, B_{k}, x_{k}, y_{k}\right]^{T}$

where $C_{k}$ is the single superpixel, composed of $R_{k}, G_{k}, B_{k}$ channels and $x_{k}, y_{k}$ coordinates. The distance of each superpixel is defined by:

$D s=d_{R G B}+\frac{m}{S} d_{x y}$

where $m$ is the compactness of a superpixel. The $d_{x y}$ is the distance in $x-y$ space which considers the coordinates of every pixel. The mathematical representation of $d_{x y}$ is given as:

$d_{x y}=\sqrt{\left(x_{k}-x_{i}\right)^{2}+\left(y_{k}-y_{i}\right)^{2}}$

In equation (6) $S$ is the distance between superpixels centers and given as

$S=\sqrt{\frac{N}{Z}}$

Similarly the $d_{R G B}$ in equation (6) is the distance in $R G B$ color space and basically a Euclidian distance between the coordinates of two pixels which is given as:

$d_{R G B}=\sqrt{\left(R_{k}-R_{i}\right)^{2}+\left(G_{k}-G_{i}\right)^{2}+\left(B_{k}-B_{i}\right)^{2}}$

From these components, we can measure every pixel's color and location weather they are similar or not. The overall concept of selecting the most intense superpixel is demonstrated in Fig. 3. Initially, the superpixel boundaries are covered on the original image. Then Equation 5 initializes cluster centers and repeat until stability for each $C_{k}$ and finds similar pixels in the neighborhood to compute new centers. After, computing the new centers of the superpixels, the mean $R G B$ color of the superpixel region has been assigned to each pixel in the output image. Further, we have computed each superpixel $R G B$ channels intensities which later compared with every superpixel intensity and selected the most intense superpixel by the designed constraint. Thus, we considered $R G B$ intensities of the highest intense superpixel as the atmospheric light parameter $A$ which automatically selects the hazy region from the input hazy image. Mathematically formulation of $\mathrm{A}$ is given as:

$A=\max \_$int $\left(C_{k}\left\{R_{k}, G_{k}, B_{k}\right\}\right)$

where max _int denotes the maximum intensity of the extracted intense superpixel for the RGB channels.

\subsection{Estimating the transmission map}

When the atmospheric light $A$ is given, the transmission $t$ can be estimated. For this purpose DCP [32] suggested that transmission in a local patch $\Omega\left(x_{g}\right)$ is constant. Therefore, the patch transmission is denoted by $t\left(x_{g}\right)$. A minimum ( $\mathrm{min}$ ) operation is applied in the local patch on the haze imaging Equation 1, and we get the following equation:

$\min _{y \in \Omega\left(x_{g}\right)}\left(I^{c}(y)\right)=t\left(x_{g}\right) \min _{y \in \Omega\left(x_{g}\right)}\left(J^{c}(y)\right)+\left(1-t\left(x_{g}\right)\right) A^{c}$

where $C$ is a color channel. Equation 11 can be further expressed as follows: 

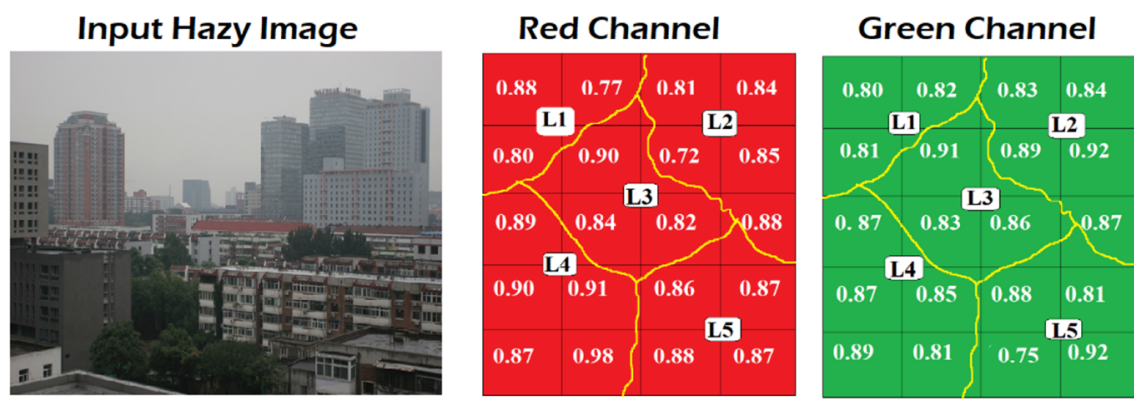

\section{Blue Channel}
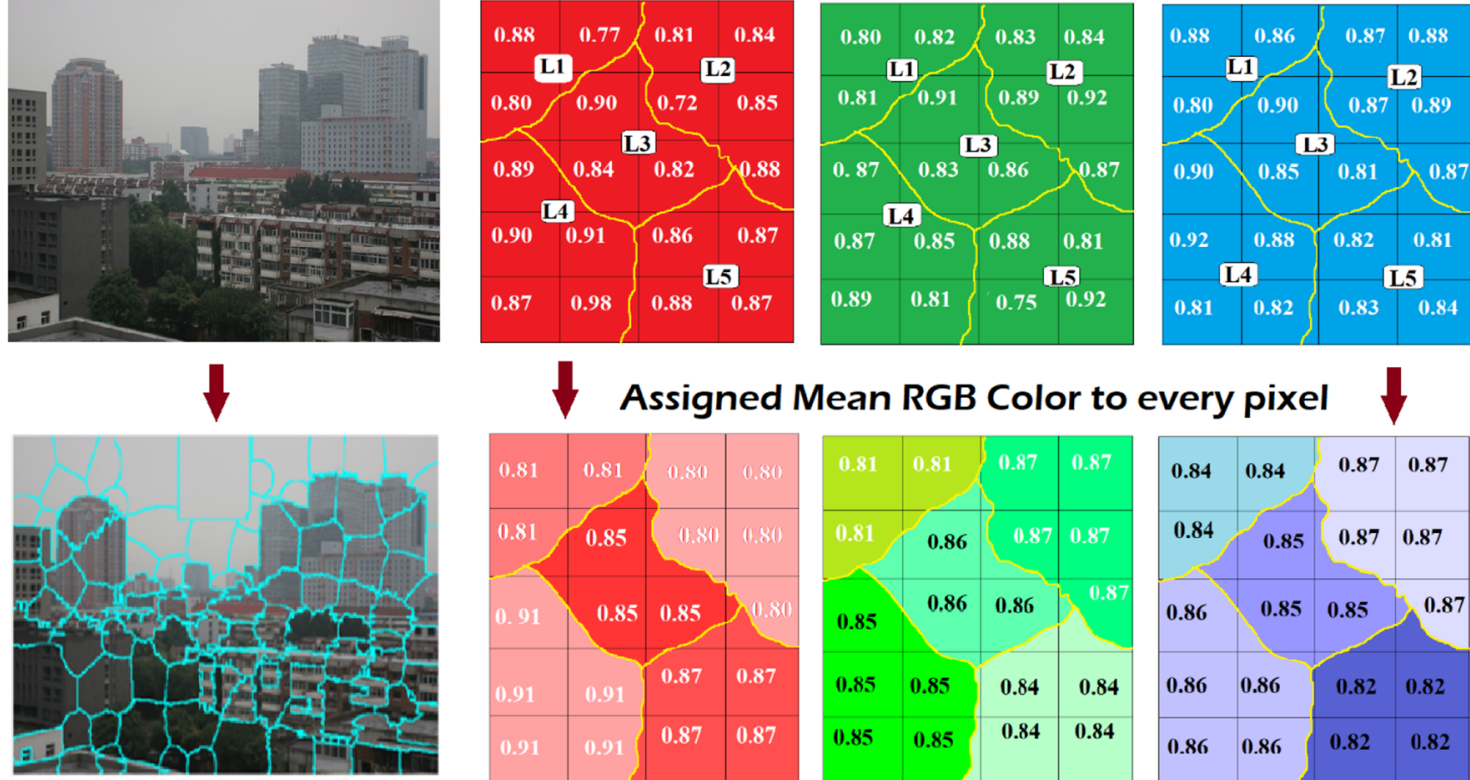

$\checkmark$ Assigned Mean RGB Color to every pixel
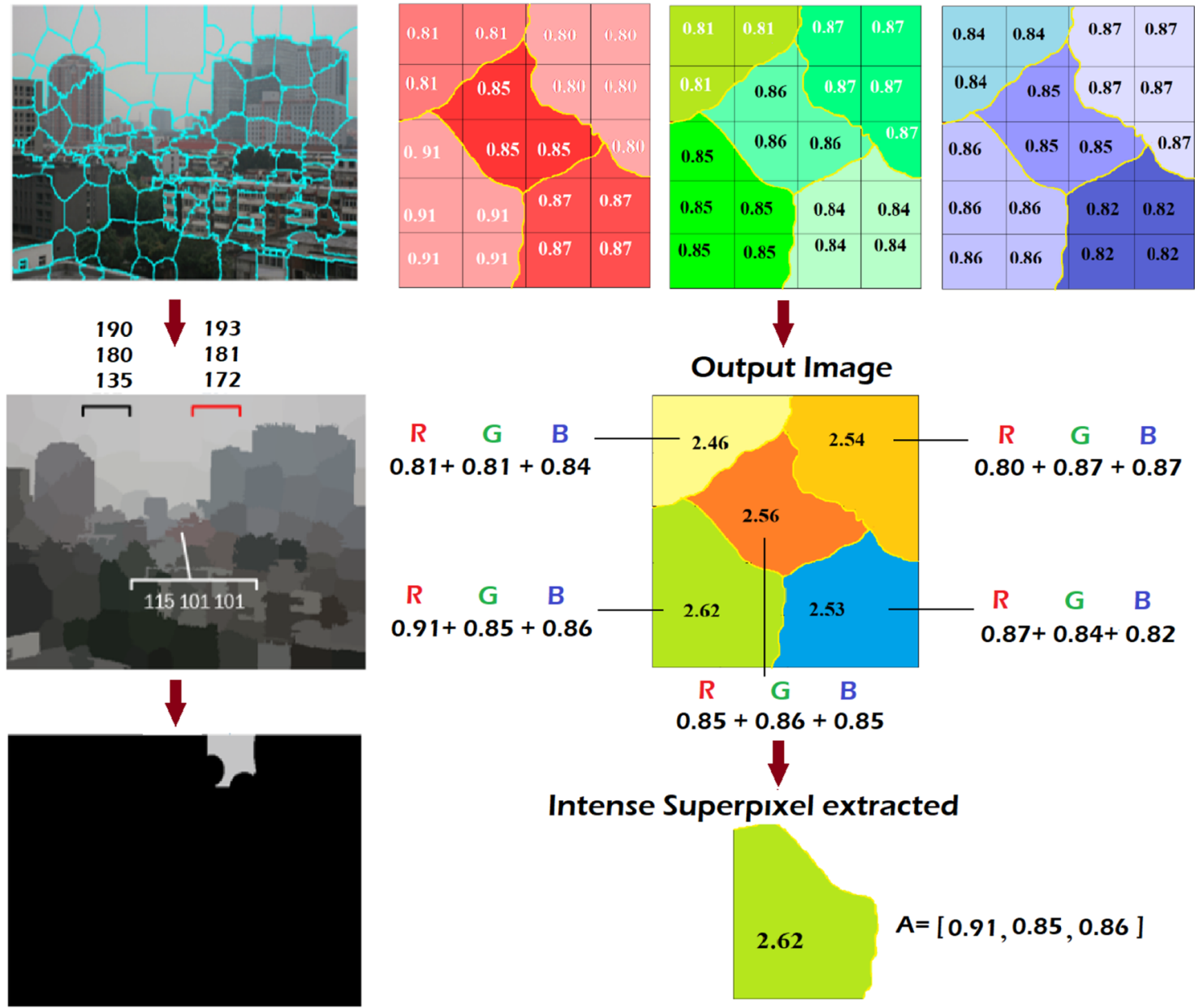

2.62

$A=[0.91,0.85,0.86]$

Fig. 3 Proposed air-light estimation model which automatically selects the hazy region by extracting the most intense superpixel from the input image

$\min _{y \in \Omega\left(x_{g}\right)}\left(\frac{I^{c}(y)}{A^{c}}\right)=t\left(x_{g}\right) \min _{y \in \Omega\left(x_{g}\right)}\left(\frac{J^{c}(y)}{A^{c}}\right)+\left(1-t\left(x_{g}\right)\right)$

Note that, the min operation applied to all channels which give us the following equation:

$$
\begin{aligned}
& \min _{c}\left(\min _{y \in \Omega\left(x_{g}\right)}\left(\frac{I^{c}(y)}{A^{c}}\right)\right)=t\left(x_{g}\right) \min _{c}\left(\min _{c \in \Omega\left(x_{g}\right)}\left(\frac{J^{c}(y)}{A^{c}}\right)\right) \\
& \quad+\left(1-t\left(x_{g}\right)\right)
\end{aligned}
$$

DCP method suggests that the dark channel $J^{\text {dark }}$ of the haze-free radiance $J$ has tendency toward zero, which can be expressed as: 
$J^{\mathrm{dark}}\left(x_{g}\right)=\min _{c}\left(\min _{y \in \Omega\left(x_{g}\right)}\left(J^{c}(y)\right)\right)=0$

Equation 14 leads to the following expression:

$\min _{c}\left(\min _{y \in \Omega\left(x_{g}\right)}\left(\frac{J^{c}(z)}{A^{c}}\right)\right)=0$

Finally, the transmission is estimated by substituting Eq. 15 in Eq. 13 which is given as:

$t\left(x_{g}\right)=1-\omega \min _{c}\left(\min _{y \in \Omega\left(x_{g}\right)}\left(\frac{I^{c}(y)}{A^{c}}\right)\right)$

where $\omega$ is the parameter to keep the naturalness of the image and to perceive the depth for the human eye.

\subsection{Transmission map refinement}

Images contain many pieces of information such as textures, structures, and edges. Many real-world applications require to remove several uninformative contents from the images; however, performing such operations on images can cause the deterioration of the image textures, structures, and edges. For instance, obtaining the transmission map for dehazing using the techniques discussed in previous sections, the parameter $\omega$ such as in Eq. 16 is supplied to keep naturalness of the dehaze output image. However, this addition may weaken the structures and edges after dehazing. Similarly, guided filtering method [42] still lacks in efficiency near to edges. For this purpose, we proposed rolling guidance filter [50]. It completely controls the details of the filtered image under a scale measure, which works iteratively and converges quickly. As an outcome, it produced artifact-free results by separating unwanted details while preserving important details of the image. The adopted rolling guidance filter [50] is a scale-aware and can refine the obtained transmission map effectively as compared to the guided filter [42].

\subsection{Problem analysis and formulation for transmission refinement}

The rolling guidance filter [50] has two main stages; the first one to remove the small structures and the second stage is about edges recovery. Initially, the structure scale is defined as the smallest Gaussian standard deviation $\sigma_{d}$ such that when $\sigma_{d}$ is applied to the transmission, the corresponding structure vanishes. This is denoted by the convolution process of the obtained transmission $t\left(x_{g}\right)$ and the Gaussian $g_{v}(x, y)$ of variance $v=\sigma_{d}^{2}$ given as:

$R_{v}=g_{v} * t\left(x_{g}\right)$ where $g_{v}(x, y)=\frac{1}{\sqrt{2 \pi v}} \exp \left(-\frac{x^{2}+y^{2}}{2 v}\right)$ and $*$ is a convolution operator. The $R_{v}$ is the result at scale $v$. The $v$ is referred to as scale parameter in scale space theory [51]. When the structure scale in image is smaller than $\sigma_{d}$, it will be removed in the result such as $R_{v}$. This analysis provides us a solid intuition to assume the $\omega$ parameter of DCP [32] and the other extra details such as a Gaussian, i.e., $g_{v}(x, y)=g_{v}$, which generated in the process of obtaining the transmission map. In [50] for small structure removal, the filter is defined as:

$\underset{\text { small_scale_removed }^{t}\left(x_{g}\right)}{t\left(x_{g}\right)(q)}$
$t(P)=\frac{1}{K_{p}} \underset{q \in \operatorname{Nig}(p)}{\sum} \exp \left(-\frac{\|p-q\|^{2}}{2 \sigma_{d}^{2}}\right)$

where $K_{p}=\sum_{q \in \operatorname{Nig}(p)} \exp \left(-\frac{\|p-q\|^{2}}{2 \sigma_{d}^{2}}\right)$ denotes the normalization, $\operatorname{Nig}(p)$ represents the neighboring pixels of $p$, where the $p$ and $q$ are the index pixel coordinates. Therefore, initially the small structures are removed where scale is smaller than $\sigma_{d}$ as claimed in space scale theory [51]. In edge recovery phase the $t\left(x_{g}\right)_{\text {small_scale_removed }}(P)$ in Equation 18 is defined as $U . U$ is iteratively updated and denoted as $U^{t+1}$ as a result in the $t^{\text {th }}$ iteration. Note that, the $U^{t+1}$ can be obtained in the form of Joint Bilateral Filter; mathematical representation is given as:

$$
\begin{gathered}
U^{t+1}(p)=\frac{1}{K_{p}} \sum_{q \in \operatorname{Nig}(p)} \exp \left(-\frac{\|p-q\|^{2}}{2 \sigma_{d}^{2}}\right. \\
\left.-\frac{\left\|U^{t}(p)-U^{t}(q)\right\|^{2}}{2 \sigma_{\text {range }}^{2}}\right)
\end{gathered}
$$

$t\left(x_{g}\right)(q)$

where $K_{p}=\sum_{q \in \operatorname{Nig}(p)} \exp \left(-\frac{\|p-q\|^{2}}{2 \sigma_{d}^{2}}-\frac{\left\|U^{t}(p)-U^{t}(q)\right\|^{2}}{2 \sigma_{\text {rangee }}{ }^{2}}\right)$ is to be considered for normalization. $\sigma_{d}$ controls the spatial and $\sigma_{r}$ controls the range weights. The described phases can be combined into a single equation by the following equation:

$U^{t+1}(p)=\frac{1}{K_{p}} \sum_{q \in \operatorname{Nig}(p)} \exp \left(-\frac{\|p-q\|^{2}}{2 \sigma_{d}^{2}}\right) t\left(x_{g}\right)(q)$

where $U^{t+1}(p)$ is a refined transmission and can be written as:

$\underset{\text { refined }}{t\left(x_{g}\right)=\frac{1}{K_{p}}} \sum_{q \in \operatorname{Nig}(p)} \exp \left(-\frac{\|p-q\|^{2}}{2 \sigma_{d}^{2}}\right) t\left(x_{g}\right)(q)$

When the parameters such as transmission $t$ and air-light $A$ are known, the scene radiance $J$ can be recovered by considering Eq. 1; we can derive the global equation for scene radiance $J$ as follows: 


\section{Input}
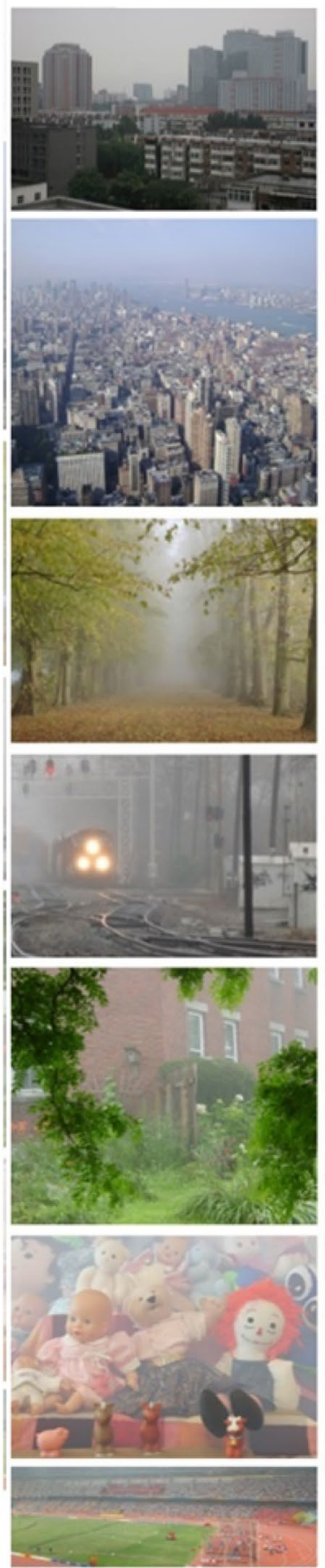

DCP [32]
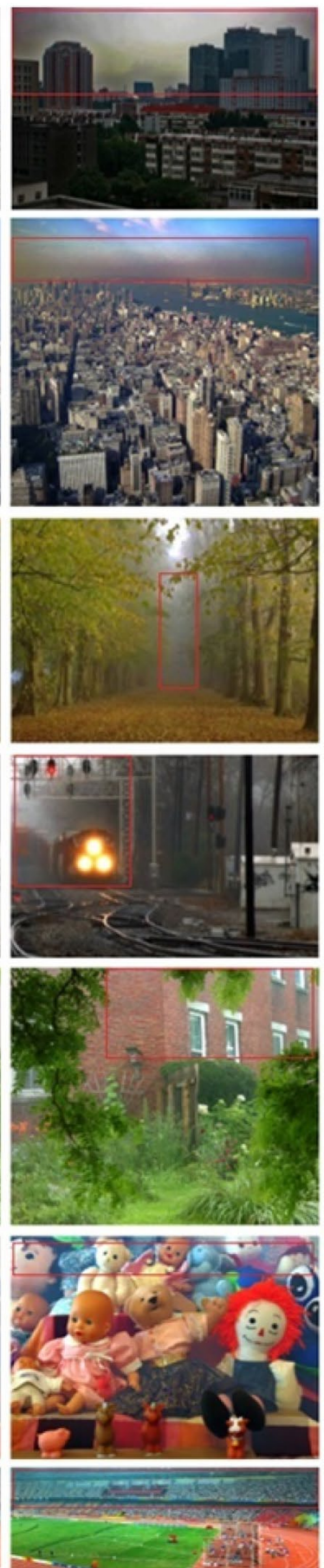

Guided Filter [42] Proposed DSMGF
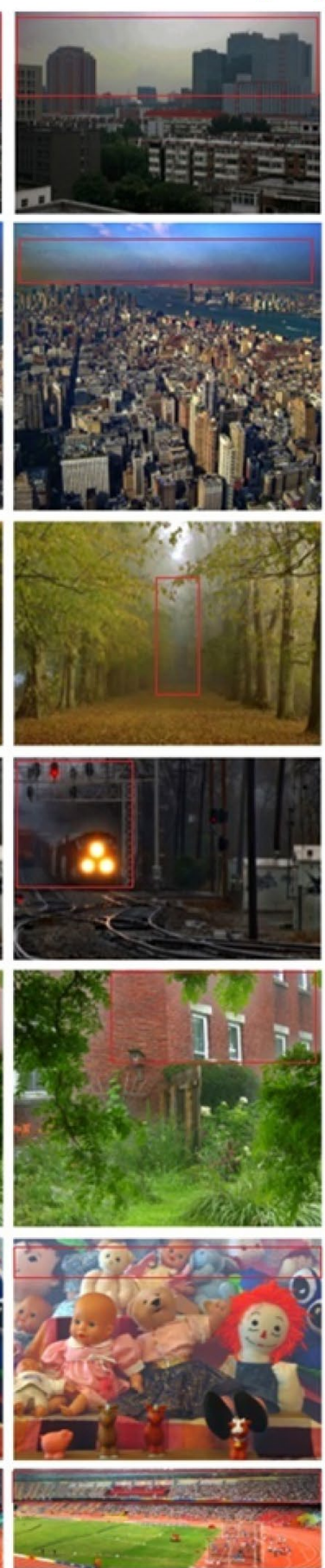
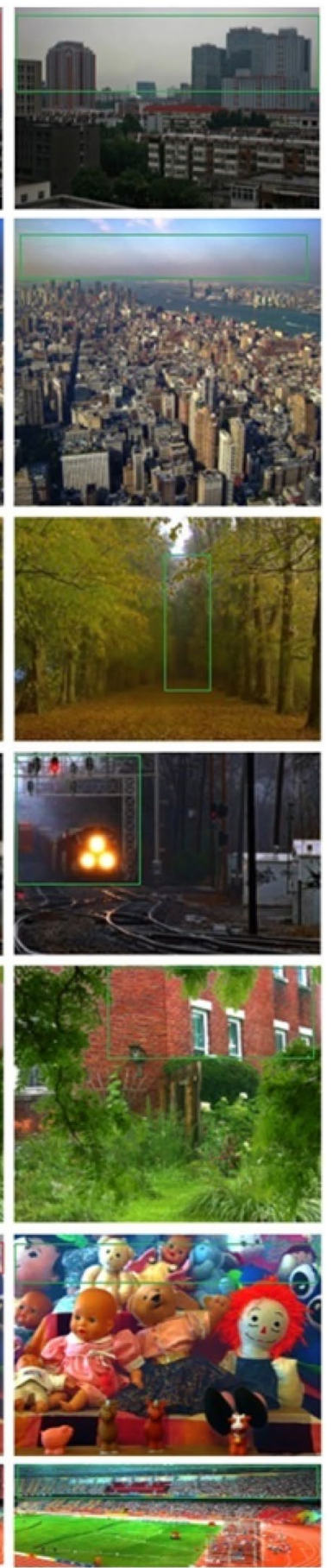

Fig. 4 Visual comparison of our proposed method with DCP [32] and Guided Filter [42]

$J\left(x_{g}\right)=\frac{I\left(x_{g}\right)-A}{t\left(x_{g}\right)}+A$

Finally, by integrating all the obtained entities into Eq. 22 can restore a haze-free image.

\section{Experimental results}

This section presents the image quality assessment based on image fidelity, i.e., subjective assessment and image readability, i.e., objective assessment. To give a clear 
(A)

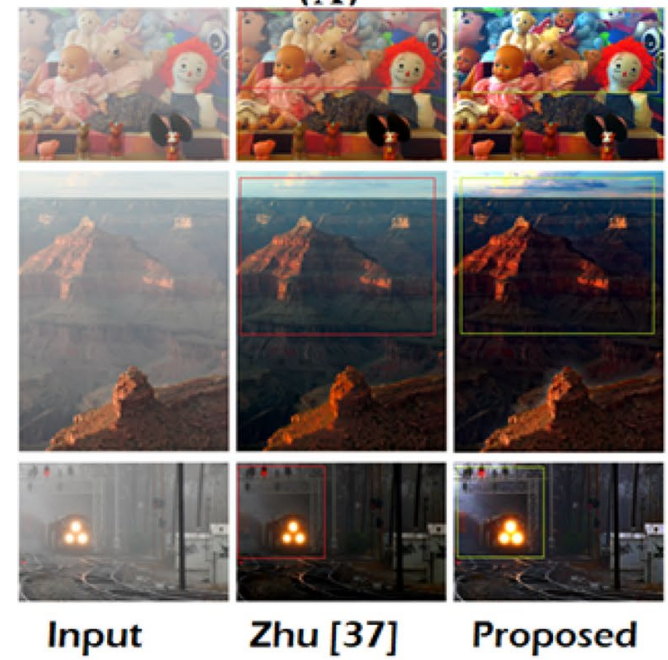

(C)

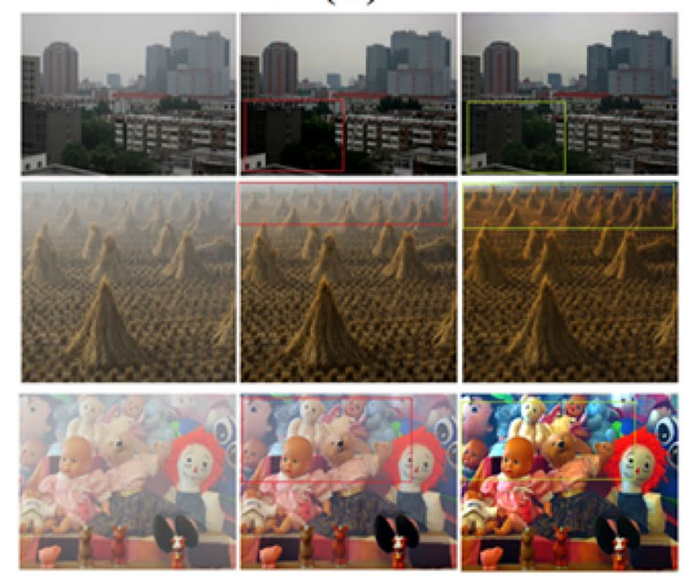

Input

Cai [54]

(E)

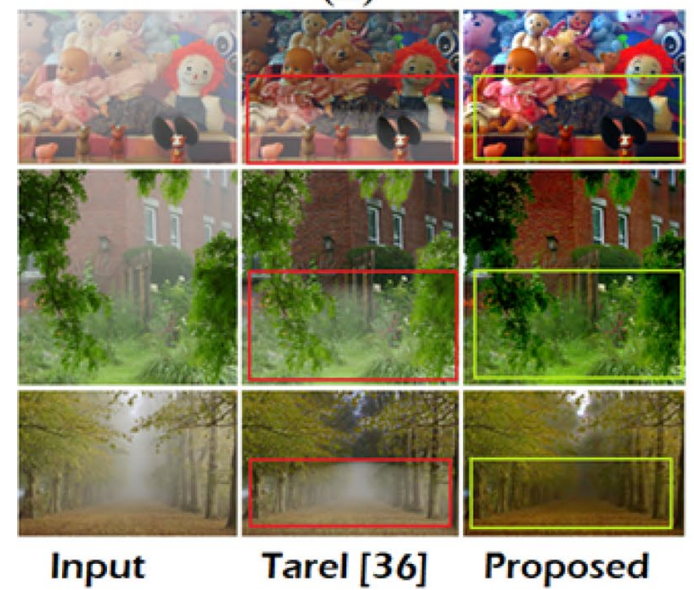

(B)
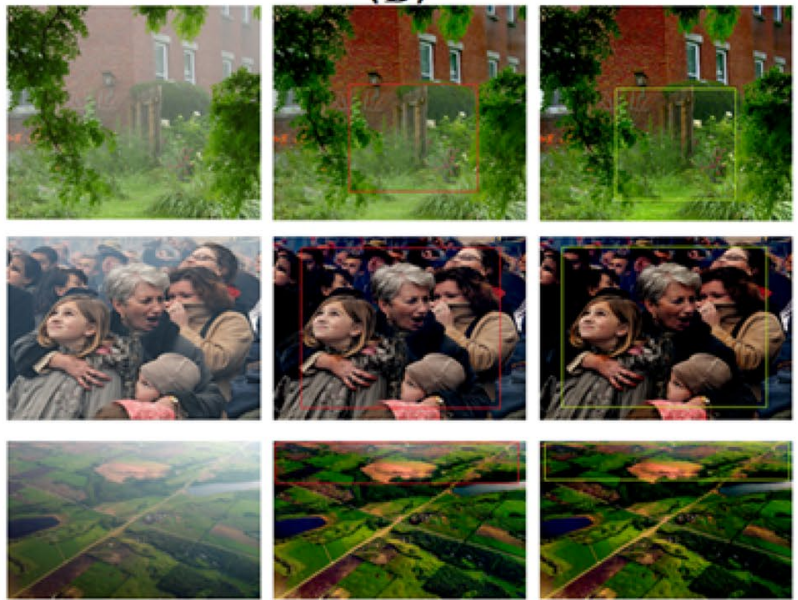

Input

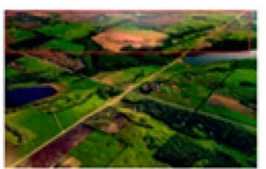

Fattal [35]

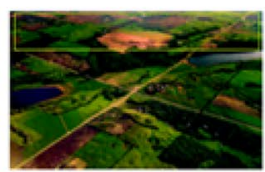

Proposed

(D)

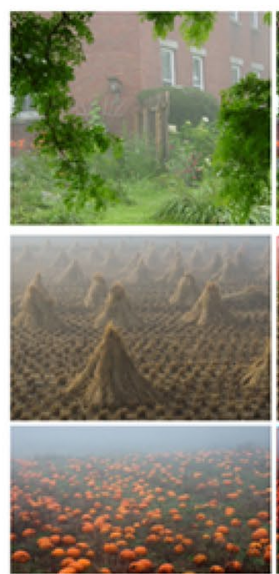

Input

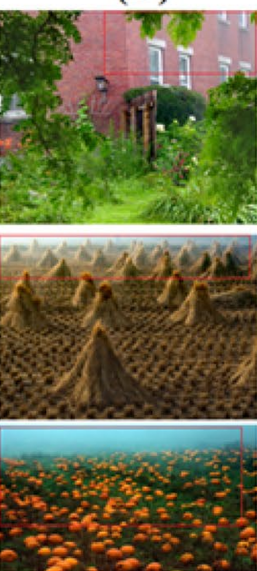

Berman [53]

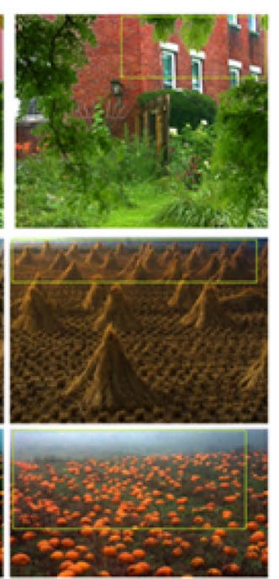

Proposed

(F)

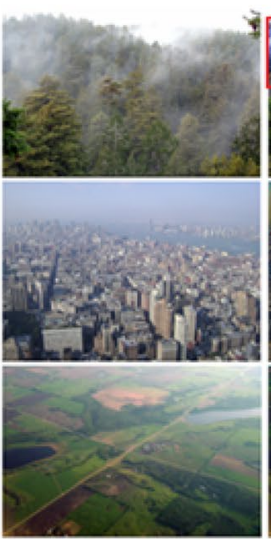

Input

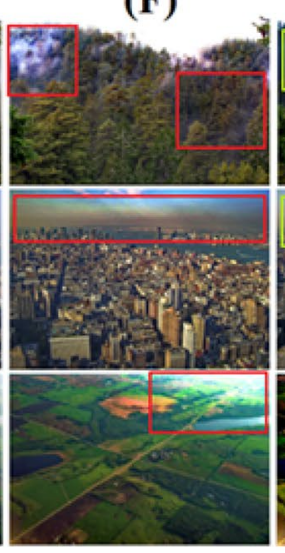

Meng [39]

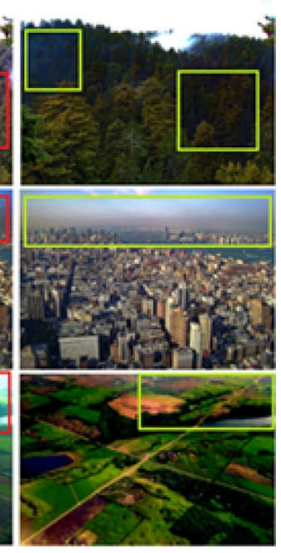

Proposed

Fig. 5 Visual comparison of the proposed method with [35-37, 39, 53, 54] on benchmark images 


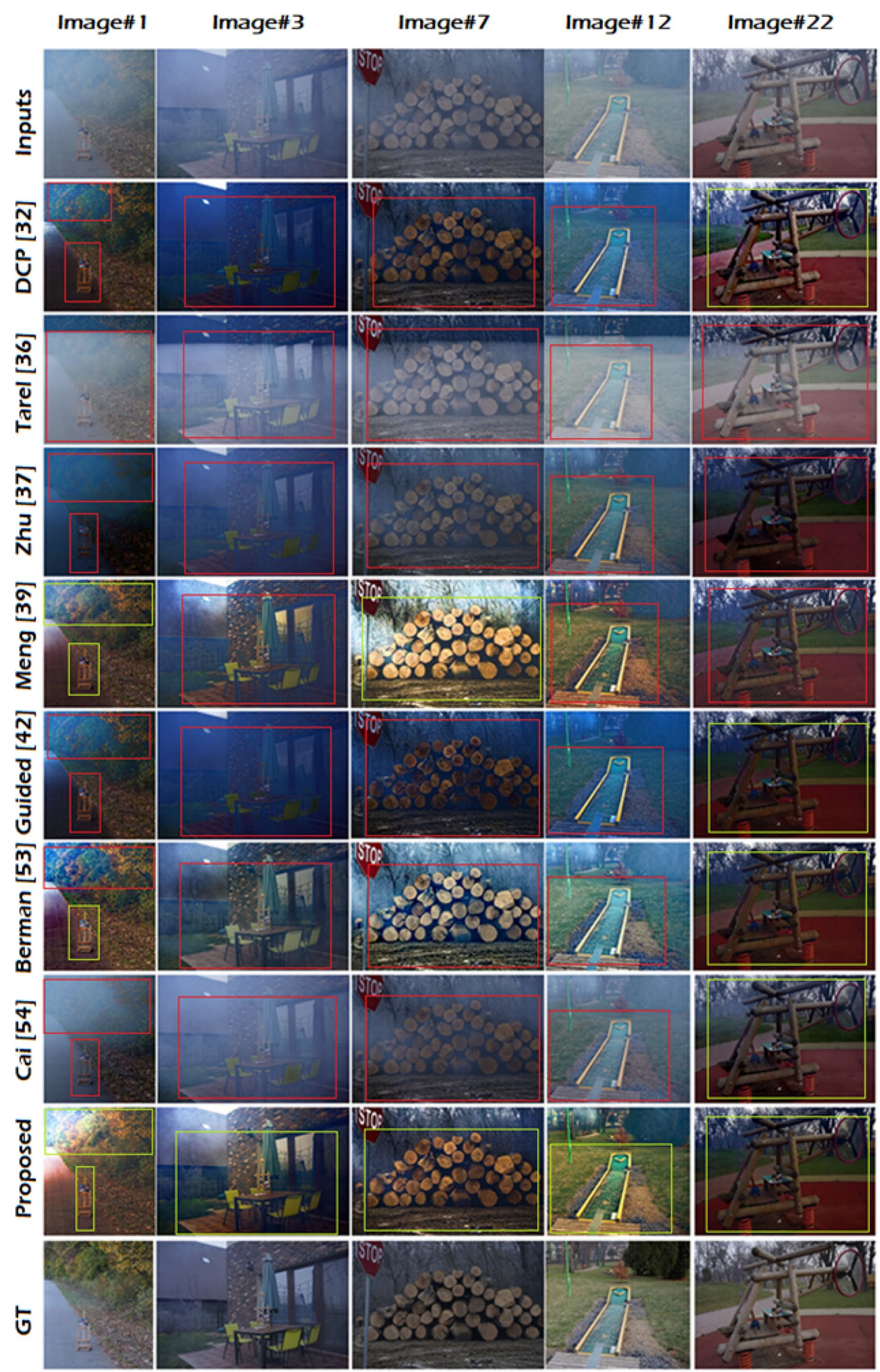

Fig. 6 Visual comparison of the proposed method with [32, 36, 37, 39, 42, 53, 54] on O-Haze Dataset [52] 


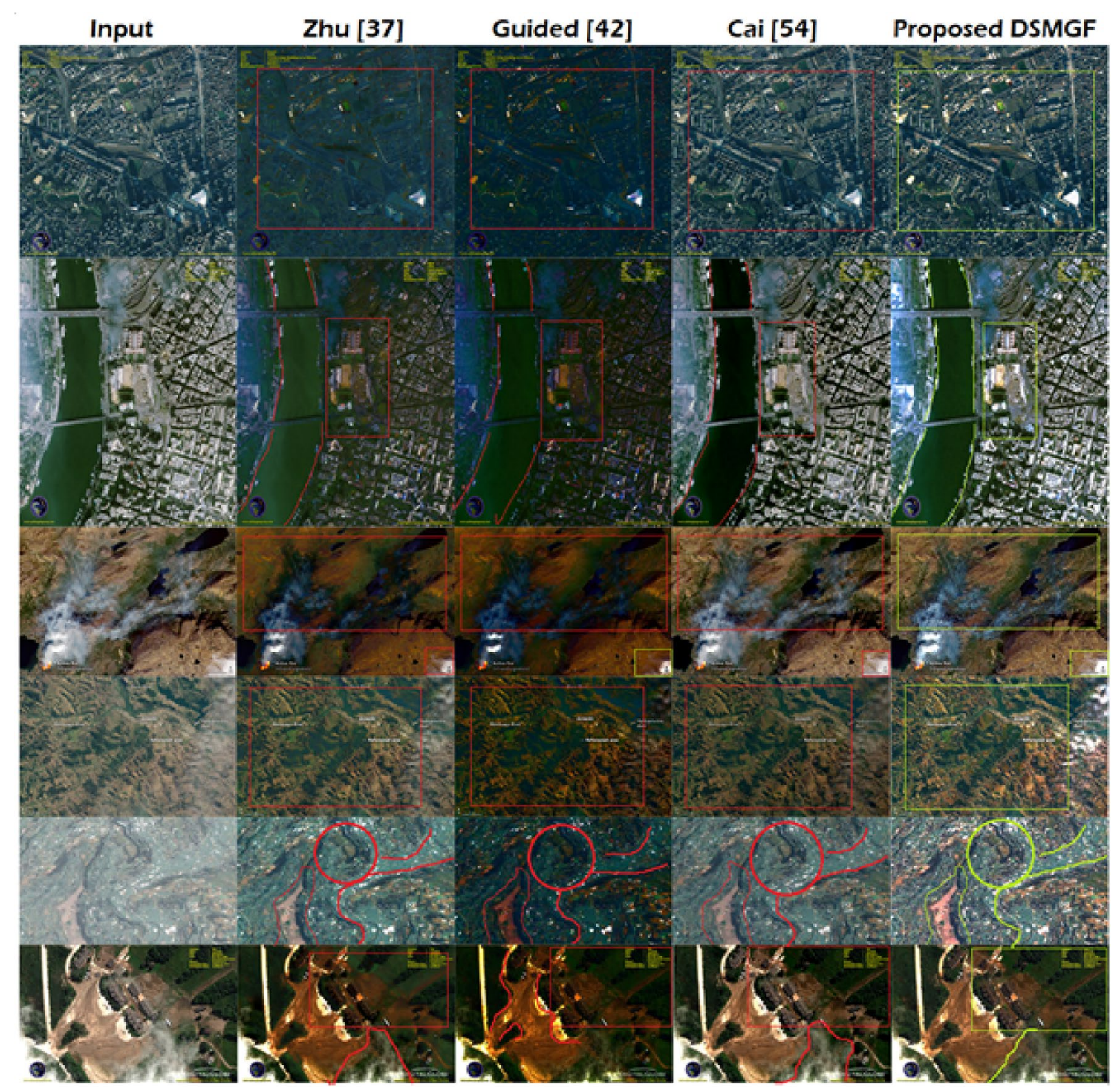

Fig. 7 Evaluation of the proposed method with $[37,42,54]$ for visual comparison on satellite imagery

intuition, we sub-categorize the experimental section as Sect. 4.1 presents the subjective assessment, Sect. 4.2 demonstrates the structures, textures, and edge-preserving characteristics, Sects. 4.3 and 4.4 show the objective assessment and computational time analysis, respectively, of our proposed method which has been tested on several datasets such as benchmark hazy images [27, 32-36, 38, 42], O-Haze [52], remotely sensed, and satellite images. All the reported results for comparison are directly generated from their online available codes. The proposed method results are promising as compared to the state-ofthe-art methods. To overcome the computational complexity and for the fair evaluation and comparisons, we have reduced the size of O-Haze images.

\subsection{Subjective assessment}

\subsubsection{Evaluation on benchmark images}

The benchmark images are equated for visual comparison with given approaches [32, 35-37, 39, 42, 53, 54]. Figure 4 shows the comparison of the proposed method with the state-of-the-art DCP method [32] and the guided filter [42]. In Fig. 4 we can clearly note the better scene radiances as outputs are recovered through our technique. For example, in image buildings, the distorted and halo artifacts are removed. In image Manhattan-1 proposed method impressively cleared the distortion in sky region while in DCP [32] and Guided Filter failed [42] to do so. 


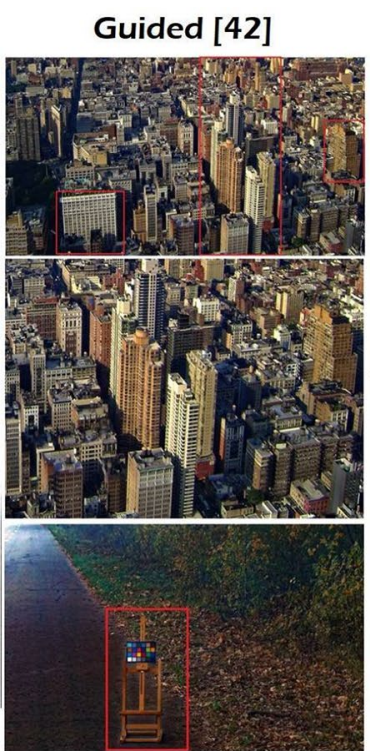

Zhu [37]
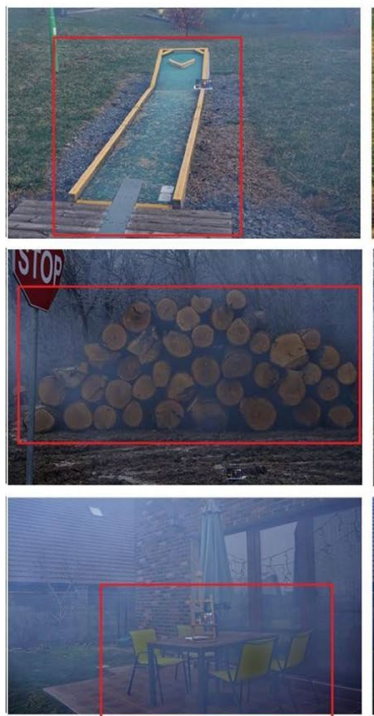
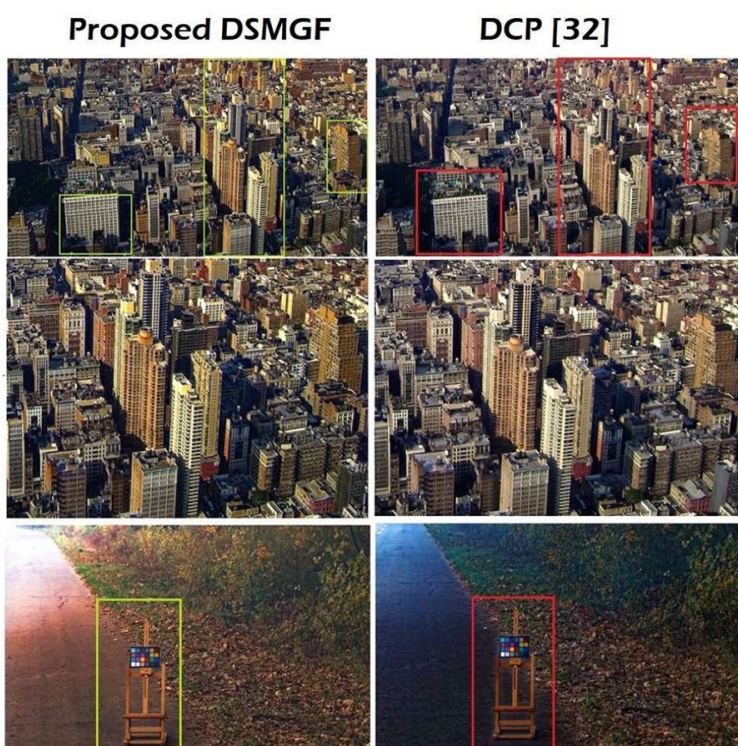

Proposed DSMGF
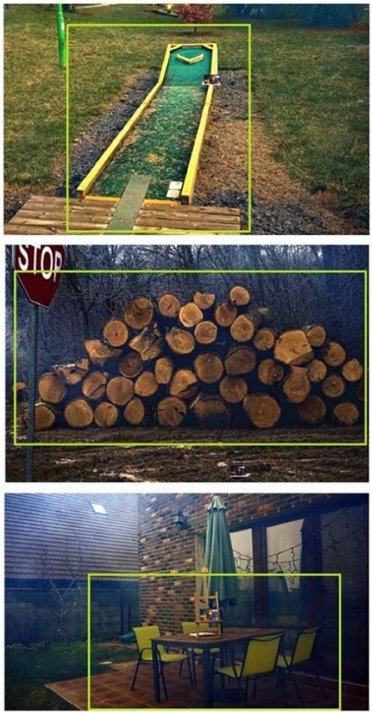

\section{Berman [53]}

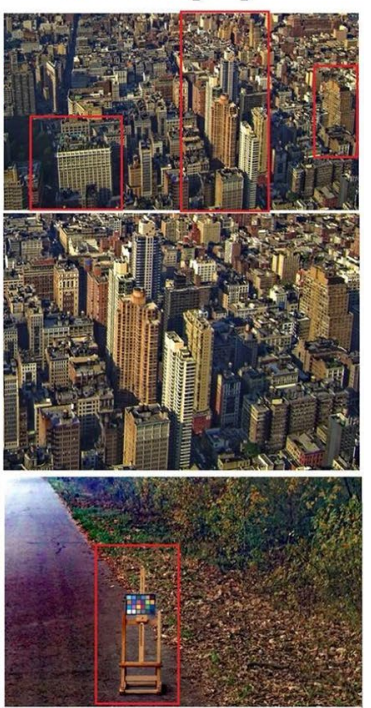

Cai [54]
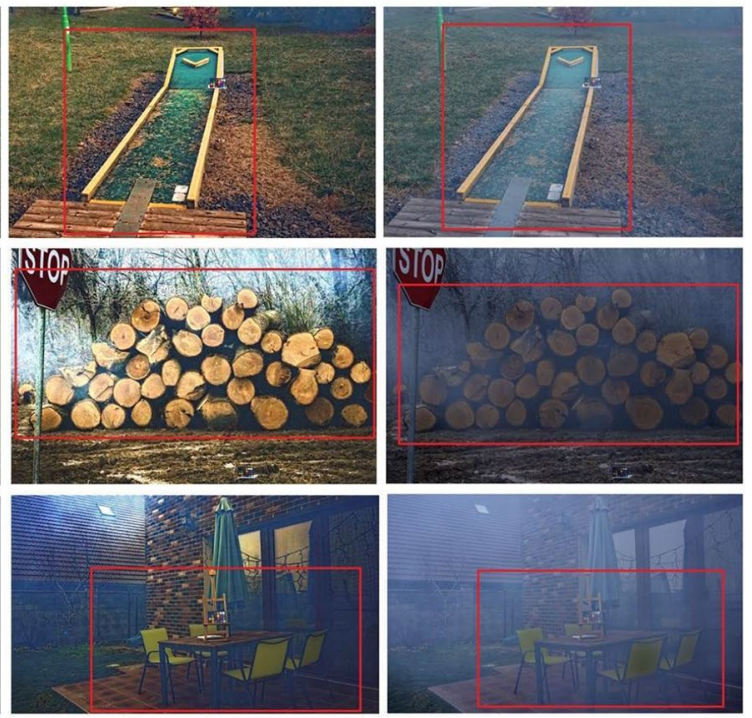

Fig. 8 Proposed method with $[32,37,39,42,53,54]$ for texture and structure analysis

Furthermore the proposed method is compared with other techniques, and their visual results are depicted in Fig. 5, where the visual superiority of the proposed method has been noted. In Fig. 5a, proposed method is compared with the method of Zhu [37] for images Dolls, Train and Rocks. The proposed method removed the haze successfully where better contrast and texture have been recovered in dehazed outputs. In Fig. 5b the Fattal [35] method dehazed the hazy images, but our method has more pleasant results in terms of its contrast and dehazing effects. For example, for the images Redbricks and Aerial, the Fattal method [35] failed to completely remove haze as compared to the proposed technique. Figure $5 \mathrm{c}$ depicts the dehaze images comparison of Cai [54] with the proposed algorithm. We can see there is still a haze in the output of Cai [54], while our method completely dehazed the hazy inputs. Figure 5d shows the comparison with Berman [53], where a lower contrast of the dehazed images has been observed as compared to our proposed method. The comparison for Tarel [36] with the proposed method is given in Fig. 5e, which has very worst performance among all of the cited methods for comparison. Although, their outputs have better contrast of some portions of the dehazed images. Yet, their generated outputs have still a very heavy hazy layers. Figure 5f shows the comparison with Meng [39]. In images Forest, Manhattan-1, and Aerial, there is still a haze in the outputs of Meng [39] method. Additionally, the 


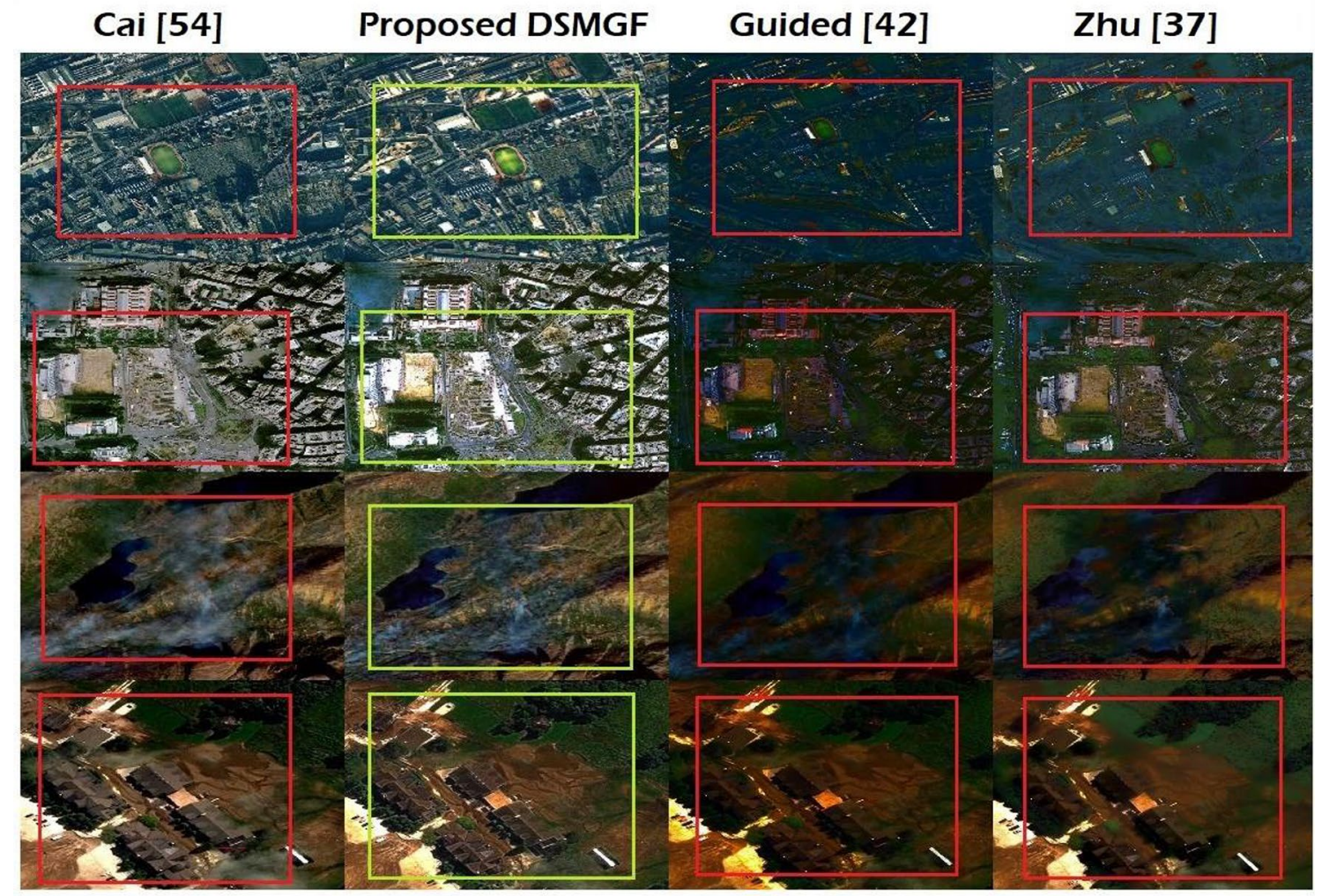

Fig. 9 Structures, textures and edges analysis on satellite imagery

over-saturation and color distortion can be seen in Manhattan-1 image dehazed by Meng [39] technique.

\subsubsection{Evaluation on 0-Haze data set}

O-Haze dataset [52] is the first outdoor scenes database composed of real hazy and corresponding ground truth images. O-Haze [52] contains different outdoor images of the different scenes depicting the haze-free (ground truth) and hazy conditions. These scenes captured under the same illumination parameters. This dataset involves investigating the existing haze in the captured scenes for scene visibility and objects radiance. To illustrate the usefulness of the proposed algorithm, O-Haze [52] is used for evaluation. We compared our proposed method with the state-of-thearts dehazing techniques such as DCP [32], Guided [42], Tarel [36], Zhu [37], Meng [39], Berman [53], and Cai [54]. Evaluating the proposed method on O-Haze data set [52], revealed quite effective results. Figure 6 depicts the comparison of our proposed algorithm with other methods.

\subsubsection{Evaluation on satellite imagery}

Nowadays, environmental pollution and fog are serious threads today's world is facing. These factors not only thread at the ground surface but also limit the performance of the satellites in the upper atmosphere. To restore a degraded image, image recoloring (image-manipulation) techniques are also important which can be used for dehazing purposes. Manipulation of digital images is rapidly growing phenomenon and a challenging task [55]. The presence of haze tends to mixed pixels in remote sensing (RS) images due to the spatial resolution of the sensor and variability of ground surface [56]. Moreover, the haze causes the degradation of visual information such as color, structures, textures, and edges [57]. Analysis of these polluted images is a challenging task for analysts and real-time applications [58]. To cope with these factors, dehazing is considered to be an important task to preprocess satellite images [59]. We observed that when there is fog, dust, aerosols, and water droplets in the atmosphere, the haze imaging model is also applicable to a passive satellite sensor (depicted in Fig. 1). In the same way, we considered hazy and foggy satellites imagery for dehazing. For this purpose, we collected some images from Geoeye-1, Landset 8, and WorldView-2 satellites http://www.satimagingcorp.com. These satellites images are evaluated on proposed method, Guided Filtering [42], Zhu method [37], and Cai method [54]. Figure 7 provides the visual evaluation comparison of the proposed method with other state-of-the-art techniques for satellite imagery. 
Table 1 Objective evaluation of benchmark hazy images

\begin{tabular}{|c|c|c|c|c|c|c|c|c|}
\hline Benchmark Images & Proposed & DCP [32] & Tarel [36] & Zhu [37] & Meng [39] & Guided [42] & Berman [53] & Cai [54] \\
\hline \multicolumn{9}{|c|}{ Mean squared error (MSE) } \\
\hline Building 1 & 1565 & 1639 & 5823 & 1625 & 1963 & 2371 & 2256 & 8621 \\
\hline WheatCones & 1471 & 2767 & 4251 & 1364 & 2037 & 2438 & 1933 & 2151 \\
\hline Manhattan-1 & 1445 & 2251 & 1635 & 4212 & 2516 & 2924 & 3165 & 2209 \\
\hline Pumpkins & 1689 & 2909 & 4913 & 1744 & 1779 & 3182 & 2708 & 1951 \\
\hline Stadium & 1310 & 1914 & 3742 & 1672 & 1478 & 2643 & 1791 & 3042 \\
\hline Dolls & 1583 & 3620 & 6697 & 2851 & 3645 & 6819 & 1744 & 3746 \\
\hline Trees & 2023 & 3671 & 2083 & 3718 & 2063 & 3111 & 3090 & 1644 \\
\hline Train & 1758 & 5792 & 1988 & 7222 & 3853 & 7953 & 4581 & 4095 \\
\hline Aerial & 1676 & 2197 & 3680 & 3600 & 1799 & 4493 & 1090 & 3763 \\
\hline Forest & 1878 & 4792 & 3498 & 5047 & 2080 & 5429 & 2733 & 2922 \\
\hline Mountain & 3632 & 7660 & 4613 & 5693 & 2876 & 8216 & 3463 & 3770 \\
\hline Red Bricks & 1403 & 1529 & 2638 & 1900 & 1576 & 1010 & 1776 & 1429 \\
\hline People & 1354 & 2305 & 2360 & 4237 & 2206 & 2137 & 1328 & 1424 \\
\hline Average & 1752.84 & 3311.23 & 3686.23 & 3452.69 & 2297.77 & 4055.85 & 2435.23 & 3135.92 \\
\hline \multicolumn{9}{|c|}{ Peak signal-to-noise ratio (PSNR) } \\
\hline Building 1 & 21.758 & 19.9841 & 10.4791 & 16.0218 & 15.2008 & 17.1045 & 14.597 & 19.3077 \\
\hline WheatCones & 24.647 & 21.7094 & 11.845 & 16.78 & 15.9895 & 14.8099 & 15.2663 & 17.5188 \\
\hline Manhattan-1 & 26.2405 & 18.6065 & 15.9938 & 11.8853 & 14.1231 & 16.1651 & 13.1261 & 18.2206 \\
\hline Pumpkins & 29.4003 & 27.4928 & 11.2166 & 15.714 & 15.627 & 14.694 & 13.8042 & 18.3464 \\
\hline Stadium & 17.9098 & 15.3104 & 12.3994 & 15.8963 & 16.4328 & 16.5872 & 15.5997 & 13.2982 \\
\hline Dolls & 19.379 & 18.5431 & 9.8714 & 13.5801 & 13.9059 & 12.1512 & 15.7131 & 15.7087 \\
\hline Trees & 24.3013 & 21.4819 & 14.9431 & 12.4273 & 14.9846 & 15.9086 & 13.2302 & 16.244 \\
\hline Train & 28.4736 & 26.4094 & 18.183 & 18.5299 & 20.2439 & 22.1002 & 11.5204 & 16.4992 \\
\hline Aerial & 37.1356 & 35.8421 & 12.4719 & 32.4707 & 15.579 & 13.3035 & 17.755 & 13.7159 \\
\hline Forest & 15.7757 & 14.0025 & 12.6925 & 11.1004 & 14.9494 & 12.5374 & 13.764 & 13.4726 \\
\hline Mountain & 25.9876 & 25.3254 & 11.4908 & 10.5771 & 13.5427 & 9.8732 & 12.7353 & 12.3667 \\
\hline Red Bricks & 26.1545 & 19.2884 & 13.9176 & 15.3426 & 16.1545 & 21.3954 & 18.5725 & 18.0041 \\
\hline People & 19.7307 & 17.8751 & 14.4012 & 11.8595 & 14.6928 & 17.0635 & 16.8982 & 16.5952 \\
\hline Average & 24.3764 & 21.6823 & 13.0696 & 15.5526 & 15.4943 & 15.6687 & 14.8140 & 16.0998 \\
\hline \multicolumn{9}{|c|}{ Structural similarity index metric (SSIM) } \\
\hline Building 1 & 0.8189 & 0.7498 & 0.647 & 0.7112 & 0.753 & 0.6748 & 0.8631 & 0.7797 \\
\hline WheatCones & 0.7995 & 0.7011 & 0.682 & 0.8321 & 0.7796 & 0.7176 & 0.7741 & 0.7686 \\
\hline Manhattan-1 & 0.8495 & 0.8454 & 0.8694 & 0.6367 & 0.829 & 0.8017 & 0.8075 & 0.8161 \\
\hline Pumpkins & 0.77 & 0.687 & 0.6926 & 0.7498 & 0.7236 & 0.767 & 0.7347 & 0.7618 \\
\hline Stadium & 0.8061 & 0.6582 & 0.7364 & 0.7372 & 0.6542 & 0.6611 & 0.7977 & 0.805 \\
\hline Dolls & 0.8177 & 0.5294 & 0.7001 & 0.7257 & 0.6176 & 0.6144 & 0.8014 & 0.7274 \\
\hline Trees & 0.8017 & 0.7686 & 0.8109 & 0.7391 & 0.8405 & 0.7367 & 0.825 & 0.8009 \\
\hline Train & 0.842 & 0.8111 & 0.7195 & 0.4241 & 0.6092 & 0.5085 & 0.79 & 0.7535 \\
\hline Aerial & 0.7352 & 0.566 & 0.7673 & 0.6624 & 0.7017 & 0.6213 & 0.6017 & 0.8084 \\
\hline Forest & 0.7719 & 0.6886 & 0.7514 & 0.5843 & 0.7406 & 0.658 & 0.6734 & 0.7159 \\
\hline Mountain & 0.7448 & 0.5134 & 0.6498 & 0.4771 & 0.3353 & 0.4533 & 0.605 & 0.5708 \\
\hline Red Bricks & 0.8688 & 0.8199 & 0.8064 & 0.811 & 0.8401 & 0.8264 & 0.821 & 0.7725 \\
\hline People & 0.7946 & 0.7018 & 0.7177 & 0.6125 & 0.7284 & 0.7554 & 0.7799 & 0.6896 \\
\hline Average & 0.8015 & 0.6954 & 0.7346 & 0.6694 & 0.7040 & 0.6766 & 0.7595 & 0.7515 \\
\hline \multicolumn{9}{|c|}{ Perception-based image quality evaluator (PIQE) no-reference image quality score } \\
\hline Building 1 & 50.9921 & 43.717 & 36.321 & 47.0342 & 39.01 & 44.8065 & 46.9845 & 44.793 \\
\hline WheatCones & 57.57 & 18.6026 & 25.5848 & 13.6222 & 28.7292 & 18.1037 & 32.9421 & 51.892 \\
\hline Manhattan-1 & 30.5713 & 29.3206 & 26.3178 & 29.2138 & 31.1695 & 29.5296 & 41.5572 & 23.3677 \\
\hline Pumpkins & 39.4539 & 25.1015 & 24.4791 & 22.7639 & 35.4232 & 24.7328 & 30.2217 & 28.5148 \\
\hline
\end{tabular}


Table 1 (continued)

\begin{tabular}{|c|c|c|c|c|c|c|c|c|}
\hline Benchmark Images & Proposed & DCP [32] & Tarel [36] & Zhu [37] & Meng [39] & Guided [42] & Berman [53] & Cai [54] \\
\hline Stadium & 39.0644 & 39.9332 & 33.2566 & 32.3864 & 40.4754 & 38.4992 & 37.548 & 23.0031 \\
\hline Dolls & 31.5962 & 38.8974 & 34.0157 & 45.519 & 34.8812 & 34.1787 & 44.3576 & 33.721 \\
\hline Trees & 43.234 & 43.6199 & 39.6557 & 19.5195 & 38.4818 & 43.1747 & 28.2405 & 36.5889 \\
\hline Train & 33.3798 & 33.0375 & 34.272 & 32.6669 & 32.5934 & 83.8418 & 35.1318 & 44.6617 \\
\hline Aerial & 36.2686 & 28.1259 & 25.6897 & 32.0187 & 25.5014 & 31.1838 & 24.6308 & 31.6728 \\
\hline Forest & 19.0384 & 13.0085 & 13.1984 & 14.2105 & 17.846 & 12.7286 & 21.6638 & 16.1871 \\
\hline Mountain & 15.8295 & 11.4277 & 26.4467 & 12.3746 & 14.7853 & 12.4105 & 25.0138 & 14.2749 \\
\hline Red Bricks & 31.242 & 28.7902 & 32.229 & 22.23 & 31.242 & 28.7902 & 33.1653 & 28.2786 \\
\hline People & 47.324 & 49.1253 & 40.2258 & 49.6883 & 40.0199 & 49.1253 & 50.5331 & 47.6318 \\
\hline Average & 36.5818 & 30.9774 & 30.1301 & 28.7113 & 31.5506 & 34.7004 & 34.7684 & 32.6605 \\
\hline
\end{tabular}

The images recovered though proposed method have better textures, structures, and edges. Besides this, our proposed method successfully remove the thin fog from the satellite images while the other techniques are failed to do so. The proposed method recovered radiances have better contrast and color. The second good performance we have observed of Cai method [54] on these satellite images. Guided Filtering [42] and Zhu [37] method produced radiances were not enough good as compared to our proposed method. Moreover, the radiances recovered by Guided Filtering [42] and Zhu method [37] were too much smoothen which of course not a good choice for real-time applications such as computer vision and remote sensing systems.

\subsection{Visual texture and structure analysis}

To keep important information such as textures, structures, and edges in the output dehazed images, the performance of rolling guidance filter is investigated. It observed that the use of the rolling guidance filter is very successful instead of the guided filter in terms of recovery of edges, structures, and textures. This is already explained in Sect. 3.4. The visual texture and structure analysis are shown in Fig. 8, where Fig. 9 shows the textures, structures and edges analysis on satellite imagery. The DCP [32], Guided Filtering [42], and Berman [53] method generated results have not such a solid textures and edges as compared to our proposed method. For O-Haze Image 01, all the state-of-the-art methods generated competitive outputs. However, we can see the edges and textures of the objects are not fully recovered. Similarly, we can see more amount of haze in their recovered outputs. The same observations can be made for O-Haze 12, such as the proposed method's generated output is comparative cleared and have more contrast and more visible in terms of textures and edges recovery. For images O-Haze 07 and O-Haze 03 , the proposed method outputs contain visible scenes and objects clarity. For instance, one can notice the chairs and table are more visible as compared to the outputs of other methods. Our proposed technique is superior due to the following facts. The proposed method uses rolling guidance filter as compared to the guided imaging filtering. The guided image filtering dependent on the joint bilateral filter uses more amount of Gaussian and can cause weakening of the textures, structures, and edges. Another important factor is the omega factor used for keeping naturalness of the dehazed output. The performance of Guided Filter [42] was worst, as its sheds color distortion and smoothen image more than required. Thus, it vanishes important structures and edges. Similarly, Zhu [37] performance was also average on this remotely sensed imagery. While Cai method [54] performed better on this remote sensing imagery as compared to Guided Filtering [42] and Zhu method [37]. The Cai method [54] dehazed outputs have also some drawbacks of smoothing image and not efficient to remove the thin haze contrary to our method performed well by restoring better contrast, edges, structures, and textures.

\subsection{Objective assessment}

Objective assessment performs a key role in the evolution of dehazing methods. There are many parameters described in the literature such as mean squared error (MSE), peak signal-to-noise ratio (PSNR), structural similarity (SSIM) and perception-based Image quality evaluator (PIQE) noreference image quality score. Table 1 shows these metrics scores for benchmark images, where Table 2 provides the metrics scores for O-Haze dataset [52] and satellite images. This quantitative analysis shows that our proposed algorithm performed well on all three datasets. In Table 1, in terms of MSE score the second-best algorithm is Meng [39], and the third one is Berman [53]. Similarly, in terms of PSNR, the DCP [32] performed well. For the SSIM score, again the Berman [53] performed well and got a higher average SSIM value. Further, we evaluated our proposed method and other methods on perception-based image quality evaluator (PIQE). Where we can see that apart from our method, the 
Table 2 Objective evaluation of O-Haze dataset [52] and satellite imagery

\begin{tabular}{|c|c|c|c|c|c|c|c|c|}
\hline O-Haze [52] & Proposed & DCP [32] & Tarel [36] & Zhu [37] & Meng [39] & Guided [42] & Berman [53] & Cai [54] \\
\hline \multicolumn{9}{|c|}{ Mean squared error (MSE) } \\
\hline Image\#1 & 2649.9797 & 3618.3543 & 2948.9352 & 5285.7755 & 2744.8863 & 4688.3609 & 5285.7755 & 4158.987 \\
\hline Image\#3 & 1748.8062 & 3190.3107 & 1499.8183 & 1979.302 & 2966.9628 & 4673.7761 & 1979.302 & 1839.4082 \\
\hline Image\#7 & 1489.0485 & 1984.9063 & 4926.6406 & 591.3968 & 4381.7134 & 2380.9107 & 949.3968 & 2562.4431 \\
\hline Image\#10 & 2012.3204 & 3058.7392 & 1717.4271 & 3468.6823 & 4185.1399 & 5222.1735 & 3468.6823 & 3612.5807 \\
\hline Image\#12 & 1860.5571 & 3874.9812 & 4183.0022 & 2698.5728 & 3652.398 & 4457.924 & 2698.5728 & 3183.0022 \\
\hline Image\#22 & 1448.782 & 3229.0557 & 3903.3843 & 4103.9785 & 1735.1656 & 4254.3936 & 4103.9785 & 2967.169 \\
\hline Image\#27 & 1816.5503 & 3255.4049 & 2172.9438 & 4187.9027 & 4085.9958 & 8034.0564 & 4187.9027 & 3979.0133 \\
\hline Average & 1860.8634 & 3173.1074 & 3050.3073 & 3187.9443 & 3393.1802 & 4815.9421 & 3239.0872 & 3186.0862 \\
\hline \multicolumn{9}{|c|}{ Peak signal-to-noise ratio (PSNR) } \\
\hline Image\#1 & 21.0006 & 20.5457 & 18.3584 & 10.8997 & 13.7456 & 11.4206 & 12.5816 & 17.49 \\
\hline Image\#3 & 26.7394 & 19.0925 & 16.3704 & 15.1657 & 14.3889 & 11.4341 & 14.1803 & 16.6879 \\
\hline Image\#7 & 28.3809 & 15.1534 & 18.4617 & 21.2342 & 11.7144 & 14.3634 & 15.9249 & 20.63 \\
\hline Image\#10 & 20.0938 & 16.2754 & 15.782 & 12.7292 & 11.9137 & 10.9523 & 13.8803 & 20.2592 \\
\hline Image\#12 & 25.6175 & 22.2481 & 17.4009 & 13.8195 & 12.505 & 11.6395 & 16.3186 & 19.1074 \\
\hline Image\#22 & 19.6113 & 13.04 & 18.8705 & 11.9988 & 15.7374 & 11.8424 & 15.5119 & 15.1924 \\
\hline Image\#27 & 21.0569 & 16.0048 & 14.7603 & 11.9108 & 12.0178 & 9.0815 & 11.8442 & 15.1663 \\
\hline Average & 23.2143 & 17.4799 & 17.1434 & 13.9654 & 13.1461 & 11.5334 & 14.3202 & 17.7904 \\
\hline \multicolumn{9}{|c|}{ Structural similarity index metric (SSIM) } \\
\hline Image\#1 & 0.6724 & 0.3023 & 0.9468 & 0.5676 & 0.1044 & 0.5387 & 0.3155 & 0.793 \\
\hline Image\#3 & 0.8902 & 0.4098 & 0.9272 & 0.8632 & 0.6056 & 0.6289 & 0.6156 & 0.8284 \\
\hline Image\#7 & 0.6805 & 0.6194 & 0.9272 & 0.9257 & 0.2931 & 0.5938 & 0.495 & 0.6075 \\
\hline Image\#10 & 0.8155 & 0.5877 & 0.9367 & 0.7386 & 0.7259 & 0.6483 & 0.6702 & 0.7452 \\
\hline Image\#12 & 0.7475 . & 0.2974 & 0.9448 & 0.8339 & 0.3836 & 0.5277 & 0.6725 & 0.7125 \\
\hline Image\#22 & 0.7308 & 0.596 & 0.9327 & 0.6 & 0.7867 & 0.5414 & 0.6356 & 0.7043 \\
\hline Image\#27 & 0.7558 & 0.4522 & 0.9097 & 0.7298 & 0.5295 & 0.4585 & 0.4773 & 0.7226 \\
\hline Average & 0.7433 & 0.4664 & 0.9321 & 0.7512 & 0.4898 & 0.5624 & 0.5545 & 0.7305 \\
\hline \multicolumn{9}{|c|}{ Perception-based image quality evaluator (PIQE) no-reference image quality score } \\
\hline Image\#1 & 34.9921 & 21.3283 & 18.73 & 21.5726 & 14.4581 & 11.811 & 27.7534 & 14.8556 \\
\hline Image\#3 & 30.5713 & 21.288 & 17.1948 & 24.5466 & 20.3637 & 19.0957 & 29.3902 & 21.1508 \\
\hline Image\#7 & 31.5962 & 17.824 & 15.9657 & 13.0883 & 15.8543 & 10.0953 & 14.2414 & 14.8871 \\
\hline Image\#10 & 26.2686 & 28.2742 & 18.8169 & 18.2987 & 27.157 & 23.7636 & 14.1081 & 18.0492 \\
\hline Image\#12 & 24.0384 & 25.1002 & 15.3707 & 13.5775 & 19.2519 & 23.2059 & 13.6475 & 19.7756 \\
\hline Image\#22 & 23.6356 & 23.7959 & 12.7742 & 22.2106 & 21.2159 & 28.7264 & 22.0496 & 12.8867 \\
\hline Image\#27 & 39.4618 & 29.883 & 27.6015 & 21.6892 & 22.2663 & 29.4618 & 36.8612 & 18.0822 \\
\hline Average & 30.0805 & 23.9276 & 18.0648 & 19.2833 & 20.0810 & 20.8799 & 22.5787 & 17.0981 \\
\hline Satellite imag & & Propos & & Zhu [3 & & Guided [42] & & Cai [54] \\
\hline \multicolumn{9}{|c|}{ Mean squared error (MSE) } \\
\hline Image\#1 & & 1272.7 & & 1850.8 & & 2797.8024 & & 1508.1568 \\
\hline Image\#2 & & 1526.6 & & 3629.6 & & 6258.2137 & & 627.6459 \\
\hline Image\#3 & & 1502.8 & & 3490.9 & & 4531.8852 & & 631.0249 \\
\hline Image\#4 & & 1033.5 & & 1004.1 & & 5197.383 & & 2473.9491 \\
\hline Image\#5 & & 2437.4 & & 2784.8 & & 13280.0194 & & 3533.9832 \\
\hline Image\#6 & & 1649.8 & & 2976.9 & & 4753.7639 & & 1972.3256 \\
\hline Average & & 1570.5 & & 2622.9 & & 6136.5112 & & 1791.1809 \\
\hline \multicolumn{9}{|c|}{ Peak signal-to-noise ratio (PSNR) } \\
\hline Image\#1 & & 25.7 & & 15.4 & & 13.6626 & & 21.2364 \\
\hline Image\#2 & & 20.9 & & 12.5 & & 10.1663 & & 20.1537 \\
\hline Image\#3 & & 19.0 & & 12.7 & & 11.568 & & 20.1303 \\
\hline
\end{tabular}


Table 2 (continued)

\begin{tabular}{|c|c|c|c|c|}
\hline Satellite imagery & Proposed & Zhu [37] & Guided [42] & Cai [54] \\
\hline Image\#4 & 18.9213 & 18.1126 & 10.973 & 14.1969 \\
\hline Image\#5 & 16.2614 & 13.6828 & 6.8988 & 12.6482 \\
\hline Image\#6 & 18.9564 & 13.3931 & 11.3604 & 16.8882 \\
\hline Average & 19.9827 & 14.3131 & 10.7715 & 17.5422 \\
\hline \multicolumn{5}{|c|}{ Structural similarity index metric (SSIM) } \\
\hline Image\#1 & 0.9634 & 0.6155 & 0.5493 & 0.9021 \\
\hline Image\#2 & 0.8762 & 0.6658 & 0.4063 & 0.7636 \\
\hline Image\#3 & 0.9059 & 0.5159 & 0.5032 & 0.8878 \\
\hline Image\#4 & 0.8086 & 0.8774 & 0.4564 & 0.7766 \\
\hline Image\#5 & 0.7619 & 0.7736 & 0.3775 & 0.8259 \\
\hline Image\#6 & 0.8508 & 0.5883 & 0.4522 & 0.7527 \\
\hline Average & 0.8611 & 0.6727 & 0.4574 & 0.8181 \\
\hline \multicolumn{5}{|c|}{ Perception-based image quality evaluator (PIQE) no-reference image quality score } \\
\hline Image\#1 & 33.9247 & 29.6709 & 24.4308 & 34.7706 \\
\hline Image\#2 & 26.0271 & 21.4251 & 18.5775 & 19.3406 \\
\hline Image\#3 & 40.5434 & 24.3906 & 14.4421 & 34.5113 \\
\hline Image\#4 & 21.5068 & 19.9712 & 22.928 & 19.9863 \\
\hline Image\#5 & 30.1163 & 22.5668 & 26.7142 & 22.6335 \\
\hline Image\#6 & 20.2408 & 17.6096 & 13.9249 & 17.3809 \\
\hline Average & 28.7265 & 22.6057 & 20.1695 & 24.7705 \\
\hline
\end{tabular}

Fig. 10 Computational analysis of benchmark images

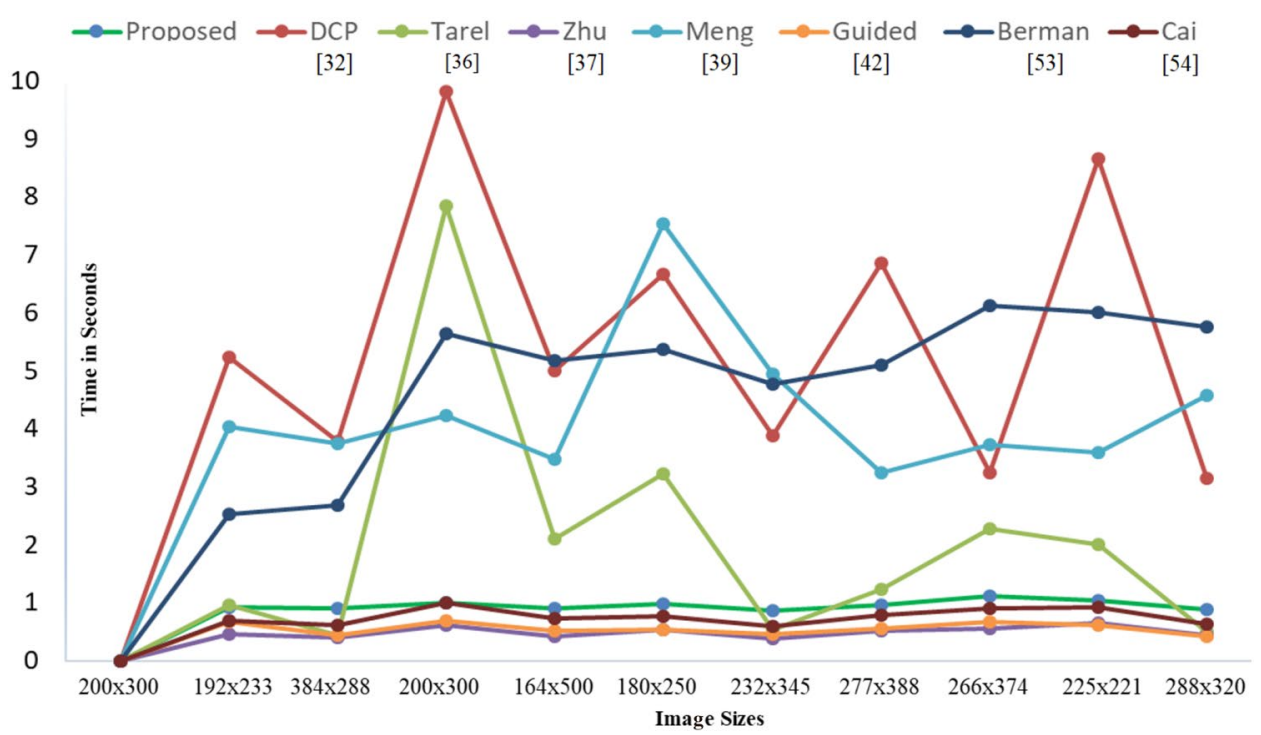

second-best performer is Berman [53] and got the maximum average PIQE score. Additionally, we extended our evaluation metrics to the O-Haze dataset [52] and remotely sensed imagery. In Table 2 we can note that the Tarel [36] method has second-best performance in terms of MSE Score for O-Haze dataset [52] while Cai [54] and DCP [32] produced better PSNR and PIQE values, respectively. In terms of SSIM, again the Tarel [36] method out-performed all the compared methods including our proposed method. For satellite imagery our evaluation is based on 4 algorithms. The second-best method which is observed in terms of MSE, PSNR, SSIM, and PIQE is the method of Cai [54]. Here, we refer the readers to respective tables which are depicting the evaluation metrics score. 


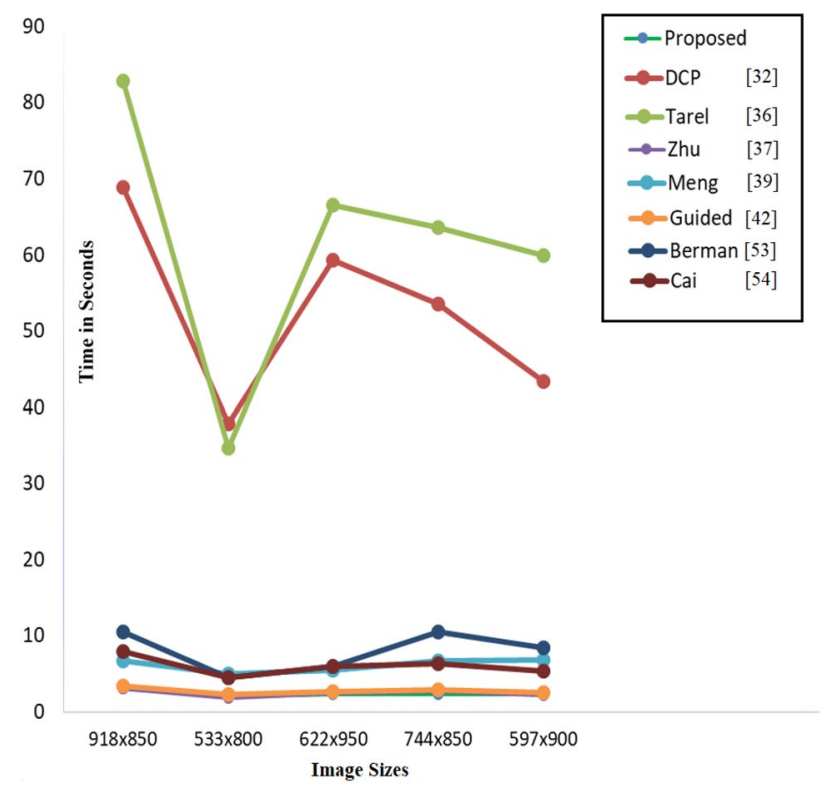

Fig. 11 Computational analysis of O-Haze dataset [52]

\subsection{Computational analysis}

For real-time image processing, the computational time of an algorithm is to be considered as an important aspect. So image and video applications need lower computational cost while extracting features from the images [60-62]. Keeping this important fact in view, we evaluated the proposed method and all others compared techniques [32, 36, 37, $39,42,53,54]$ on the benchmark images, O-Haze dataset [52], and remote sensed imagery. For our experimentation, the system specification is ASUS machine with Intel Core i7-6700HQ 2.60 GHz CPU running with installed memory (RAM) of $8.00 \mathrm{~GB}$, with MATLAB 2016b under windows 10. On benchmark images the performance of the proposed method is better than the methods of DCP [32], Tarel [36], Meng [39], and Berman [53], while Zhu [53], Guided [42] and Cai [54] methods are faster with respect to some fractions of a second. However, the performance of Zhu [37], Guided [42], and Cai [54] becomes slower when the image

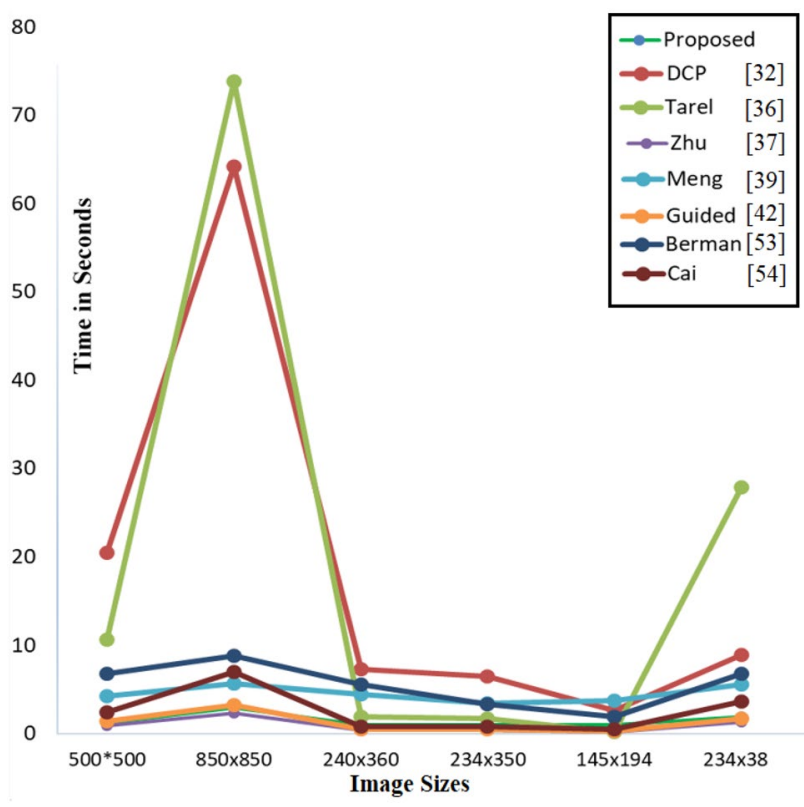

Fig. 12 Computational analysis of remote sensed imagery

dimensions get higher. For instance, evaluating the highdimensional O-Haze datasets [52] images the proposed method processing time is fast as compared with Zhu [37], Guided [42], and Cai [54]. The computational evaluation is provided in the respective Figs. 10, 11 and 12.

\subsection{Validation of the air-light estimation model}

To validate our proposed method for air-light estimation model, we performed statistical analysis on approximately 500 hazy images, downloaded from different online sources including the dataset images, to see whether the proposed algorithm is selecting the right hazy area from the input haze image or not. Because the atmospheric light is an important parameter for recovering the haze-free image, in Table 3 we noted that when there are bigger regions of brighter objects in the hazy input image, the proposed model may lead us to inaccurate atmospheric light value. To solve this, we

Table 3 A statistical analysis that shows the divergence of the proposed atmospheric light model using the superpixels segmentation method

\begin{tabular}{llllll}
\hline No. of images & $\begin{array}{l}\text { Bright objects/regions in } \\
\text { input hazy image }\end{array}$ & $\begin{array}{l}\text { No of super- } \\
\text { pixels }\end{array}$ & Haze amount & $\begin{array}{l}\text { Accurate hazy region extrac- } \\
\text { tion (air-light value) }\end{array}$ & $\begin{array}{l}\text { False hazy region } \\
\text { extraction (air-light } \\
\text { value) }\end{array}$ \\
\hline 100 & Normal & 100 & As in input image & Yes & No \\
100 & Slightly increased & 100 & As in input image & Yes & No \\
100 & Further increased & 100 & As in input image & Yes/no & Yes/no \\
100 & Further increased & 100 & As in input image & No & Yes \\
\hline
\end{tabular}

Creating smaller superpixels (by increasing number of superpixels) with respect to increased brighter objects/regions of an input hazy image may extract false hazy area/region from the input hazy image 
Table 4 A statistical analysis that shows the accuracy of the proposed atmospheric light model using the superpixels segmentation method

\begin{tabular}{llllll}
\hline No. of images & $\begin{array}{l}\text { Bright objects/regions in } \\
\text { input hazy image }\end{array}$ & $\begin{array}{l}\text { No of super- } \\
\text { pixels }\end{array}$ & Haze amount & $\begin{array}{l}\text { Accurate hazy region extrac- } \\
\text { tion (air-light value) }\end{array}$ & $\begin{array}{l}\text { False hazy region } \\
\text { extraction (air-light } \\
\text { value) }\end{array}$ \\
\hline 100 & Normal & 100 & As in input image & Yes & No \\
100 & Slightly increased & 80 & As in input image & Yes & No \\
100 & Further increased & 60 & As in input image & Yes & No \\
100 & Further increased & 40 & As in input image & Yes & No \\
\hline
\end{tabular}

Creating bigger superpixels (by reducing the number of superpixels) with respect to increased brighter objects/regions of the input hazy image can extract the right hazy area/region from the input hazy image

reduced the number of superpixels when there are increase in brighter objects/regions in the input hazy image. Thus, it will estimate the optimal atmospheric light value which is shown in Table 4. Therefore, we have come across this trade off by creating bigger superpixels to extract right atmospheric light value.

\section{Conclusions}

This work proposed a novel two-stage image dehazing and defogging method. In the first stage, this work adopts a superpixels segmentation technique to segment the hazy image into superpixels to account the atmospheric light values. At the second stage, we proposed the use of a rolling guidance filter to refine the initial transmission instead of the guided filter. Rolling guidance filter preserves better textures, structures, and edges in the dehazed image irrespective of previous works and produces remarkable results. The proposed method is evaluated both subjectively and objectively on benchmark hazy images, O-Haze challenging dataset and satellite images. The experimental evaluation reveals that the proposed method outperforms the state-of-the-art methods. The proposed method can be useful for real-time applications such as video-guided transportation, outdoor surveillance's, the auto-driver backed systems and remote sensing due to its fast processing of higher-dimensional images. The proposed work can further be extended to underwater image enhancement and images captured in snow, rain, and fog. Our future research direction entails validating the proposed method on hazy videos and the development of hierarchal deep models.

\section{Compliance with ethical standards}

Conflict of interest The authors declare that they have no conflict of interest.

\section{References}

1. Koschmieder, H.: Theorie der horizontalen sichtweite. Beitrage zur Physik der freien Atmosphare, pp. 33-53 (1924)

2. Shehata, M.S., Cai, J., Badawy, W.M., Burr, T.W., Pervez, M.S., Johannesson, R.J., Radmanesh, A.: Video-based automatic incident detection for smart roads: the outdoor environmental challenges regarding false alarms. IEEE Trans. Intell. Transp. Syst. 9(2), 349-360 (2008)

3. Bronte, S., Bergasa, L. M., Alcantarilla, P. F.: Fog detection system based on computer vision techniques. In: 2009 12th International IEEE Conference on Intelligent Transportation Systems, pp. 1-6, IEEE

4. Huang, S.-C., Chen, B.-H., Cheng, Y.-J.: An efficient visibility enhancement algorithm for road scenes captured by intelligent transportation systems. IEEE Trans. Intell. Transp. Syst. 15(5), 2321-2332 (2014)

5. Huang, S.-C.: An advanced motion detection algorithm with video quality analysis for video surveillance systems. IEEE Trans. Circuits Syst. Video Technol. 21(1), 1-14 (2010)

6. Jia, Z., Wang, H., Caballero, R.E., Xiong, Z., Zhao, J., Finn, A.: A two-step approach to see-through bad weather for surveillance video quality enhancement. Mach. Vis. Appl. 23(6), 1059-1082 (2012)

7. Wang, W., Yuan, X.: Recent advances in image dehazing. IEEE/ CAA J. Autom. Sin. 4(3), 410-436 (2017)

8. Jiang, H., Lu, N.: Multi-scale residual convolutional neural network for haze removal of remote sensing images. Remote Sens. 10(6), 945 (2018)

9. Shen, Y., Wang, Y., Lv, H., Qian, J.: Removal of thin clouds in landsat-8 oli data with independent component analysis. Remote Sens. 7(9), 11481-11500 (2015)

10. Sun, L., Latifovic, R., Pouliot, D.: Haze removal based on a fully automated and improved haze optimized transformation for landsat imagery over land. Remote Sens. 9(10), 972 (2017)

11. Ahmad, M., Khan, A. M., Hussain, R., Protasov, S., Chow, F., Khattak, A. M.: Unsupervised geometrical feature learning from hyperspectral data. In: 2016 IEEE Symposium Series on Computational Intelligence (SSCI), pp. 1-6 (2016)

12. Pavlic, M., Rigoll, G., Ilic, S.: Classification of images in fog and fog-free scenes for use in vehicles. In: 2013 IEEE Intelligent Vehicles Symposium (IV), pp. 481-486, IEEE

13. Spinneker, R., Koch, C., Park, S.-B., Yoon, J. J.: Fast fog detection for camera based advanced driver assistance systems. In: 17th International IEEE Conference on Intelligent Transportation Systems (ITSC), pp. 1369-1374, IEEE 
14. Negru, M., Nedevschi, S., Peter, R.I.: Exponential contrast restoration in fog conditions for driving assistance. IEEE Trans. Intell. Transp. Syst. 16(4), 2257-2268 (2015)

15. Kim, T.K., Paik, J.K., Kang, B.S.: Contrast enhancement system using spatially adaptive histogram equalization with temporal filtering. IEEE Trans. Consum. Electron. 44(1), 82-87 (1998)

16. Dippel, S., Stahl, M., Wiemker, R., Blaffert, T.: Multiscale contrast enhancement for radiographies: Laplacian pyramid versus fast wavelet transform. IEEE Trans. Med. Imaging 21(4), 343-353 (2002)

17. Cooper, T.J., Baqai, F.A.: Analysis and extensions of the franklemccann retinex algorithm. J. Electron. Imaging 13(1), 85-93 (2004)

18. Seow, M.-J., Asari, V.K.: Ratio rule and homomorphic filter for enhancement of digital colour image. Neurocomputing 69(7-9), 954-958 (2006)

19. Hautiere, N., Tarel, J.-P., Aubert, D.: Towards fog-free in-vehicle vision systems through contrast restoration. In: IEEE Conference on Computer Vision and Pattern Recognition, pp. 1-8, Citeseer (2007)

20. Kopf, J., Neubert, B., Chen, B., Cohen, M., Cohen-Or, D., Deussen, O., Uyttendaele, M., Lischinski, D.: Deep photo: modelbased photograph enhancement and viewing, vol. 27. ACM (2008)

21. Narasimhan, S. G., Nayar, S. K.: Interactive (de) weathering of an image using physical models. In: IEEE Workshop on color and photometric Methods in computer Vision, vol. 6, p. 1, France

22. Nayar, S. K., Narasimhan, S. G.: Vision in bad weather. In: Proceedings of the Seventh IEEE International Conference on Computer Vision, vol. 2, pp. 820-827, IEEE

23. Schechner, Y. Y., Narasimhan, S. G., Nayar, S. K.: Instant dehazing of images using polarization. In: CVPR (1), pp. 325-332

24. Tian, Y., Xiao, C., Chen, X., Yang, D., Chen, Z.: Haze removal of single remote sensing image by combining dark channel prior with superpixel. Electron. Imaging 2016(2), 1-6 (2016)

25. Oakley, J.P., Satherley, B.L.: Improving image quality in poor visibility conditions using a physical model for contrast degradation. IEEE Trans. Image Process. 7(2), 167-179 (1998)

26. Ancuti, C.O., Ancuti, C.: Single image dehazing by multi-scale fusion. IEEE Trans. Image Process. 22(8), 3271-3282 (2013)

27. Kratz, L., Nishino, K.: Factorizing scene albedo and depth from a single foggy image. In: IEEE 12th International Conference on Computer Vision, pp. 1701-1708, IEEE (2009)

28. Wang, Z., Feng, Y.: Fast single haze image enhancement. Comput. Electr. Eng. 40(3), 785-795 (2014)

29. Shwartz, S., Namer, E., Schechner, Y. Y.: Blind haze separation. In: IEEE Computer Society Conference on Computer Vision and Pattern Recognition (CVPR'06), vol. 2, pp. 1984-1991, IEEE (2006)

30. Tavallali, P., Yazdi, M., Khosravi, M.R.: Robust cascaded skin detector based on adaboost. Multimed. Tools Appl. 78(2), 2599-2620 (2019)

31. Narasimhan, S.G., Nayar, S.K.: Contrast restoration of weather degraded images. IEEE Trans. Pattern Anal. Mach. Intell. 6, 713-724 (2003)

32. He, K., Sun, J., Tang, X.: Single image haze removal using dark channel prior. IEEE Trans. Pattern Anal. Mach. Intell. 33(12), 2341-2353 (2010)

33. Tan, R. T.: Visibility in bad weather from a single image. In: IEEE Conference on Computer Vision and Pattern Recognition, pp. 1-8, IEEE (2008)

34. Tripathi, A., Mukhopadhyay, S.: Single image fog removal using anisotropic diffusion. IET Image Process. 6(7), 966-975 (2012)

35. Fattal, R.: Single image dehazing. ACM Trans. Graph. 27(3), $72(2008)$
36. Tarel, J.-P., Hautiere, N.: Fast visibility restoration from a single color or gray level image. In: IEEE 12th International Conference on Computer Vision, pp. 2201-2208, IEEE (2009)

37. Zhu, Q., Mai, J., Shao, L.: A fast single image haze removal algorithm using color attenuation prior. IEEE Trans. Image Process. 24(11), 3522-3533 (2015)

38. Fattal, R.: Dehazing using color-lines. ACM Trans. Graph. 34(1), 13 (2014)

39. Meng, G., Wang, Y., Duan, J., Xiang, S., Pan, C.: Efficient image dehazing with boundary constraint and contextual regularization. In: Proceedings of the IEEE International Conference on Computer Vision, pp. 617-624

40. Kim, J.-H., Jang, W.-D., Sim, J.-Y., Kim, C.-S.: Optimized contrast enhancement for real-time image and video dehazing. J. Vis. Commun. Image Represent. 24(3), 410-425 (2013)

41. Gibson, K. B., Nguyen, T. Q.: Fast single image fog removal using the adaptive wiener filter. In: IEEE International Conference on Image Processing, pp. 714-718, IEEE (2013)

42. He, K., Sun, J., Tang, X.: Guided image filtering. IEEE Trans. Pattern Anal. Mach. Intell. 35(6), 1397-1409 (2012)

43. McCartney, E.J.: Optics of the Atmosphere: Scattering by Molecules and Particles, p. 421. Wiley, New York (1976)

44. Preetham, A.J., Shirley, P., Smits, B.: A practical analytic model for daylight. In: Proceedings of the 26th Annual Conference on Computer Graphics and Interactive Techniques, pp. 91-100 (1999)

45. Abbasi, R., Luo, B., Rehman, G., Hassan, H., Iqbal, M.S., Xu, L.: A new multilevel reversible bit-planes data hiding technique based on histogram shifting of efficient compressed domain. Vietnam J. Comput. Sci. 5(2), 185-196 (2018)

46. Abbasi, R., et al.: Efficient lossless compression based reversible data hiding using multilayered n-bit localization. Secur. Commun. Netw. 2019, 8981240 (2019)

47. Hassan, H., Bashir, A.K., Abbasi, R., Ahmad, W., Luo, B.: Single image defocus estimation by modified Gaussian function. Trans. Emerg. Telecommun. Technol. 30(6), e3611 (2019)

48. Wang, W., Chang, F., Ji, T., Wu, X.: A fast single-image dehazing method based on a physical model and gray projection. IEEE Access 6, 5641-5653 (2018)

49. Achanta, R., Shaji, A., Smith, K., Lucchi, A., Fua, P., Susstrunk, S.: Slic superpixels compared to state-of-the-art superpixel methods. IEEE Trans. Pattern Anal. Mach. Intell. 34(11), 2274-2282 (2012)

50. Zhang, Q., Shen, X., Xu, L., Jia, J.: Rolling guidance filter. In: European conference on computer vision, pp. 815-830, Springer

51. Lindeberg, T.: Scale-space theory: a basic tool for analyzing structures at different scales. J. Appl. Stat. 21(1-2), 225-270 (1994)

52. Ancuti, C. O., Ancuti, C., Timofte, R., De Vleeschouwer, C.: O-haze: a dehazing benchmark with real hazy and haze-free outdoor images. In: Proceedings of the IEEE Conference on Computer Vision and Pattern Recognition Workshops, pp. 754-762

53. Berman, D., Avidan, S.: Non-local image dehazing. In: Proceedings of the IEEE Conference on Computer Vision and Pattern Recognition, pp. 1674-1682

54. Cai, B., Xu, X., Jia, K., Qing, C., Tao, D.: Dehazenet: an end-toend system for single image haze removal. IEEE Trans. Image Process. 25(11), 5187-5198 (2016)

55. Al Sharif, S., Al Ali, M., Al Reqabi, N., Iqbal, F., Baker, T., Marrington, A.: Magec: an image searching tool for detecting forged images in forensic investigation. In: 2016 8th IFIP International Conference on New Technologies, Mobility and Security (NTMS), pp. 1-6, IEEE (2016)

56. Ahmad, M., Bashir, A.K., Khan, A.M.: Metric similarity regularizer to enhance pixel similarity performance for hyperspectral unmixing. Optik 140, 86-95 (2017) 
57. Singh, D., Kumar, V.: Dehazing of remote sensing images using improved restoration model based dark channel prior. Imaging Sci. J. 65(5), 282-292 (2017)

58. Tahir, Z., Qureshi, A.H., Ayaz, Y., Nawaz, R.: Potentially guided bidirectionalized RRT* for fast optimal path planning in cluttered environments. Robot. Auton. Syst. 108, 13-27 (2018)

59. Ju, M., Ding, C., Guo, Y.J., Zhang, D.: Remote sensing image haze removal using gamma-correction-based dehazing model. IEEE Access 7, 5250-5261 (2018)

60. Abdulhussain, S. H., Ramli, A. R., Mahmmod, B. M., Saripan, M. I., Al-Haddad, S., Baker, T., Flayyih, W. N., Jassim, W. A.: A fast feature extraction algorithm for image and video processing. In: 2019 International Joint Conference on Neural Networks (IJCNN), pp. 1-8, IEEE (2019)

61. Jain, D.K., et al.: An efficient and adaptable multimedia system for converting PAL to VGA in real-time video processing. J. RealTime Image Process. (2019). https://doi.org/10.1007/s11554-01900889-4

62. Ayyappan, S., Lakshmi, C., Menon, V.: A secure reversible data hiding and encryption system for embedding EPR in medical images. J. Curr. Signal Transduct. Ther. 14, 1 (2019). https://doi. org/10.2174/1574362414666190304162411

Publisher's Note Springer Nature remains neutral with regard to jurisdictional claims in published maps and institutional affiliations.

Haseeb Hassan received his master's degree in 2012, in Computer Sciences from International Islamic University, Islamabad, Pakistan. Currently, he is enrolled in Ph.D. Computer Science in the School of Computer Science and Technology, Anhui University, Hefei, China. His area of research interests is in Computer Vision, Image Processing, and Machine Learning.

Ali Kashif Bashir is a Senior Lecturer at the Department of Computing and Mathematics, Manchester Metropolitan University, United Kingdom. He is also holding Adjunct Professor position at National University of Science and Technology, Pakistan. He is a senior member of IEEE, invited member of IEEE Industrial Electronic Society, member of ACM, and Distinguished Speaker of ACM. His past assignments include Associate Professor of ICT, University of the Faroe Islands, Denmark; Osaka University, Japan; Nara National College of Technology, Japan; the National Fusion Research Institute, South Korea; Southern Power Company Ltd., South Korea, and the Seoul Metropolitan Government, South Korea. He has worked on several research and industrial projects of South Korean, Japanese and European agencies and Government Ministries. He is also advising several start-ups in the field of STEM based education, block chain, robotics, and smart homes. He received his Ph.D. in computer science and engineering from Korea University, South Korea. He has authored over 100 research articles and is supervising/co-supervising several graduate $(\mathrm{MS}$ and $\mathrm{PhD})$ students. His research interests include internet of things, wireless networks, distributed systems, network/cyber security, cloud/network function virtualization, etc. He is serving as the Editorin-chief of the IEEE Future Directions Newsletter.

Muhammad Ahmad is an assistant professor at the Department of Computer Engineering, Khwaja Freed University of Engineering and Information Technology, Pakistan. Muhammad is associated with the research group of Advanced Image Processing Research Lab (AIPRL), a first Hyperspectral imaging lab in Pakistan. Muhammad is also associated with the University of Messina, Messina, Italy, as a research fellow. Muhammad authored a number of research papers in reputed journals and conferences. He is a regular reviewer for a number of top tier journals and conferences. His current research interests include Machine Learning, Computer Vision, Remote Sensing, Hyperspectral Imaging, and Wearable Computing.

Varun G. Menon is working as an Associate Professor in the Department of Computer Science and Engineering, SCMS School of Engineering and Technology, Kerala, India, from 01-01-2018. He worked as Assistant Professor in Department of Computer Science and Engineering, SCMS School of Engineering and Technology, Kerala, India, from 04-06-2012 to 31-12-2017. He performed his duties as an Assistant Professor in Department of Computer Science and Engineering, M.E.T'S School of Engineering and Technology, Kerala, India, from 01-02-2012 to 31-05-2012 and worked as Software Developer in Global Allies Ltd, Kerala, India, from 12-05-2008 to 10-07-2009. His research interests are Internet of Things, Underwater Sensor Networks, Fog Computing and Networking, Wireless Networks, Routing Protocols, Research Publication and Ethics, Predatory and Hijacked Journals, Opportunistic and Geographic Routing, Internet of Medical Things, Application of Computer Science in Psychology, Cyber Psychology, Youth Addiction to Social Media and Internet, Communication in Disaster and Emergency Situations, Computer Enabled Learning Methodologies, ICT in Education, Educational Psychology, Training and Development, Evaluation methods in Education.

Imran Uddin Afridi recently graduated from the Department of Computer Science, COMSATS University Islamabad. His current research interests are Computer Vision, Image Processing, and Machine Learning.

Raheel Nawaz is Director of Department of Operations, Technology, Events and Hospitality Management, Manchester Metropolitan University, UK. His research areas of interest are Machine Learning, Image Processing, Technology Enhanced Learning, Applied Artificial Intelligence, Text Mining, Data Mining, Natural Language Processing, Biomedical Applications, and Biomedical Analysis.

Bin Luo received his BEng. and MEng. degrees in electronics from Anhui university, China. In 2002, he was awarded the $\mathrm{PhD}$ degree in Computer Science from the University of York, UK. He is currently a full professor at Anhui University. He is the chair of IEEE Hefei Subsection, and an associate chair of IAPR TC15. He serves as the editorin-chief of the Journal of Anhui University (Natural Science Edition), an associate editor of several international journals, including Pattern Recognition, Pattern Recognition Letters, Cognitive Computation and International Journal of Automation and Computing. He was the guest editors for the Journal Special Issue of the Pattern Recognition Letters and Cognitive Computation. His current research interests include pattern recognition and digital image processing. In particular, he is interested in structural pattern recognition, graph spectral analysis, image and graph matching. Some of his papers were published in the journals of IEEE TPAMI, IEEE TIP, Pattern Recognition, Pattern Recognition Letters and Neurocomputing, and the conferences of CVPR, NIPS, IJCAI and AAAI. 